\title{
Computational issues in large strain elasto-plasticity: an algorithm for mixed hardening and plastic spin
}

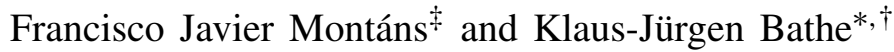 \\ Department of Mechanical Engineering, Massachusetts Institute of Technology, \\ 77 Massachusetts Avenue, Cambridge, U.S.A.
}

\begin{abstract}
SUMMARY
In this paper an algorithm for large strain elasto-plasticity with isotropic hyperelasticity based on the multiplicative decomposition is formulated. The algorithm includes a (possible) constitutive equation for the plastic spin and mixed hardening in which the principal stress and principal backstress directions are not necessarily preserved. It is shown that if the principal trial stress directions are preserved during the plastic flow (as assumed in some algorithms) a plastic spin is inadvertently introduced for the kinematic/mixed hardening case. If the formulation is performed in the principal stress space, a rotation of the backstress is inadvertently introduced as well. The consistent linearization of the algorithm is also addressed in detail. Copyright (C) 2005 John Wiley \& Sons, Ltd.
\end{abstract}

KEY WORDS: large strains; computational plasticity; plastic spin; kinematic hardening; cyclic plasticity; logarithmic strains

\section{INTRODUCTION}

Almost up to the 1990s, the continuum formulation of plasticity was based on the prescription of hypoelastic constitutive relations, the additive decomposition of the strains into an elastic and a plastic part and the use of, for example, the incremental Jaumann formulation, see e.g. References [1-3]. Nonetheless the finite element analysis of hyperelastic materials and elasto-plastic materials based on hyperelasticity was well establish 10 years earlier [4]. The use

\footnotetext{
*Correspondence to: K. J. Bathe, Department of Mechanical Engineering, Massachusetts Institute of Technology, 77 Massachusetts Avenue, Cambridge, U.S.A.

${ }^{\dagger}$ E-mail: kjb@mit.edu

¥On leave from Universidad de Castilla-La Mancha, Escuela Técnica Superior de Ingenieros Industriales, Campus Universitario s/n, E13071 Ciudad Real, Spain.

Contract/grant sponsor: Secretaría de Estado de Educación y Universidades, Ministerio de Educación, Cultura y Deportes of Spain; contract/grant number: PR2003-0345

Contract/grant sponsor: Secretaría de Estado de Política Científica y Tecnológica, Ministerio de Ciencia y Tecnología of Spain; contract/grant number: DPI2002-02065
}

Received 5 January 2004

Revised 9 July 2004

Copyright (c) 2005 John Wiley \& Sons, Ltd.

Accepted 8 November 2004 
of hypoelastic constitutive relations with a Jaumann formulation produces dissipation under elastic closed cycles [5], which is inconsistent with the definition of an elastic deformation path. It has also been found that the use of the additive decomposition of the strain tensor in finite deformation inelasticity may produce dependence of incremental elastic deformations on the deformation history [6]. Nonetheless, new corrected formulations using similar approaches have been recently advocated due to their simplicity and broad application $[7,8]$ and reported numerical simulations using isotropic plasticity seem to produce results similar to multiplicative plasticity. The approaches given in Reference [8] are based on an additive decomposition of total (instead of incremental) logarithmic strains and, going back to the work of Green and Nagdhi, the plastic logarithmic strain is defined in terms of the plastic metric tensor, considered as an internal variable.

On the other hand, multiplicative plasticity based on the so-called multiplicative (or Lee) decomposition [9] (see also Reference [10] and an earlier contribution in Reference [11]) is physically well grounded, since it is based on micromechanical dislocation theory and observations, see for example References [12-15] and the references therein. Even more, it has also a clear continuum interpretation as shown below. Therefore, computational plasticity based on this decomposition has gained popularity in the late 1980s and 1990s, see for example References [16-26] among others, although multiplicative plasticity had already been used earlier in computational mechanics [27]. The initial formulations required ad-hoc algorithms to integrate the stress-point constitutive equations due to the inherent non-linear nature of both the elastic deformation and the plastic flow [13]. More recent formulations have been developed based on a Kirchhoff hyperelastic stress function linear in terms of the logarithmic strain measures, see Reference [20] for isotropic plasticity and Reference [21] for combined hardening, see also References [22, 24-26, 28, 29], and the therein references. This hyperelastic stress function has been shown to yield excellent predictions in moderately large elastic strain ranges which develop in metal plasticity $[29,30]$. Furthermore, the natural additive property of principal logarithmic strain measures and the use of an exponential mapping in the integration of the plastic gradient allows for a simple framework, in which the small strain radial return algorithms remain unaltered and the large strain formulation reduces to a simple pre- and post-processor [21].

In this paper the formulation of Eterović and Bathe [21], see also Gabriel and Bathe [31], based on a vanishing plastic spin, is extended for the use of a (possible) constitutive equation for the plastic spin. The new formulation allows for a comparison and more detailed analysis of algorithms formulated in the principal stress space [22] and in which the principal stress directions are kept constant during the plastic flow even in the kinematic hardening case. It is shown that keeping the stress directions constant during the plastic flow introduces inadvertently a spin which obviously affects the solution results. This spin can be interpreted as a plastic spin since it only affects the plastic correction phase. Furthermore, a more detailed analysis of an algorithm in which the stress integration is performed in the principal stretch directions shows that in this case a rotation of the backstress is introduced which affects the plastic correction phase in a similar way. Hence in both these cases, different computational results are obtained from using an algorithm performed in the full stress space which does not include any plastic spin and in which the principal directions are not necessarily preserved.

Also, the consistent algorithmic tangent is calculated. In this formulation, the customary small strains consistent tangent moduli are modified to account for the non-linear geometry of the large strain formulation. 


\section{CONTINUUM FORMULATION}

In the derivation of the continuum formulation as well as in the algorithm for the stress calculation and the consistent tangent evaluation we follow the notation of References $[32,33]$.

\subsection{Multiplicative decomposition and strain rate tensors}

Let ${ }^{t} \mathbf{x}$ denote the current co-ordinates of the point ${ }^{0} \mathbf{x}$ in the undeformed configuration. The deformation gradient tensor is defined as

$$
{ }_{0}^{t} \mathbf{X}=\partial{ }^{t} \mathbf{x} / \partial^{0} \mathbf{x}
$$

The multiplicative plasticity is based on the well-known Lee decomposition [9] of the deformation gradient into an elastic and a plastic part as

$$
{ }_{0}^{t} \mathbf{X}={ }_{0}^{t} \mathbf{X}_{0}^{\mathrm{e}}{ }_{0}^{t} \mathbf{X}^{\mathrm{p}}
$$

or conceptually

$$
{ }_{0}^{t} \mathbf{X}={ }_{\tau}^{t} \mathbf{X}_{0}^{\mathrm{e} \tau} \mathbf{X}^{\mathrm{p}}
$$

The physical meaning, based on crystal plasticity, is that a unique intermediate (unloaded and here also rigid-body motion free) local configuration $\tau$ is established. It is important to recognize that this intermediate configuration is the only one with direct access to both independent elastic and plastic deformation measures and therefore represents an effective configuration to develop the continuum formulation and perform the stress-point integration. Of course as noted for example in References [15,34], an arbitrary rotation (or even deformation) may always be inserted in (2) to obtain for example ${ }_{0}^{t} \mathbf{X}={ }_{0}^{t} \mathbf{X}^{\mathrm{e}} \mathbf{Q} \mathbf{Q}^{\mathrm{T}}{ }_{0}^{t} \mathbf{X}^{\mathrm{p}}$, but since $\mathbf{Q}^{\mathrm{T}}$ does not represent a plastic deformation ${ }_{0}^{t} \mathbf{X}^{\mathrm{p}+}:=\mathbf{Q}^{\mathrm{T}}{ }_{0}^{t} \mathbf{X}^{\mathrm{p}}$ cannot be considered as a pure plastic deformation gradient as a consequence of slip mechanisms [15]. It would be somewhat awkward to have a change of the plastic gradient without any plastic deformation, with an essentially elastic behaviour and/or rigid body motion, although inclusion of the rigid body motions in the plastic deformation gradient tensor has been explored in the literature. However, if the integration algorithm is objective the stress results must be the same regardless of the preferred option.

Note also that the decomposition equation (2) is continuum based. Consider the current co-ordinates ${ }^{t} \mathbf{x}$ of a point given by

$$
{ }^{t} \mathbf{x}={ }^{0} \mathbf{x}+{ }^{t} \mathbf{u}^{\mathrm{p}}+{ }^{t} \mathbf{u}^{\mathrm{e}}
$$

where ${ }^{t} \mathbf{u}^{\mathrm{p}}$ are the 'plastic displacements' from the reference configuration to the conceptual configuration, that is due to the plastic deformations caused by slip mechanisms in the continuum (dissipative, irrecoverable) and ${ }^{t} \mathbf{u}^{\mathrm{e}}$ are the displacements from the conceptual configuration to the final (spatial) configuration due to elastic deformations and the rigid body motions in the continuum (non-dissipative and in principle recoverable). Defining ${ }^{\tau} \mathbf{x}^{\mathrm{p}} \equiv{ }^{t} \mathbf{x}^{\mathrm{p}}:={ }^{0} \mathbf{x}+{ }^{t} \mathbf{u}^{\mathrm{p}}$ the co-ordinates of a stress-free (and rigid body motion free) configuration, the gradient of (4) with 
respect to the reference configuration given by the co-ordinates ${ }^{0} \mathbf{x}$ is

$$
\begin{aligned}
{ }_{0}^{t} \mathbf{X} & =\mathbf{I}+\frac{\partial^{t} \mathbf{u}^{\mathrm{e}}}{\partial^{\tau} \mathbf{x}^{\mathrm{p}}} \frac{{ }^{\tau} \mathbf{x}^{\mathrm{p}}}{\partial^{0} \mathbf{x}}+\frac{\partial^{t} \mathbf{u}^{\mathrm{p}}}{\partial^{0} \mathbf{x}}=\mathbf{I}+\frac{\partial^{t} \mathbf{u}^{\mathrm{e}}}{\partial^{\tau} \mathbf{x}^{\mathrm{p}}}\left(\mathbf{I}+\frac{\partial^{t} \mathbf{u}^{\mathrm{p}}}{\partial^{0} \mathbf{x}}\right)+\frac{\partial^{t} \mathbf{u}^{\mathrm{p}}}{\partial^{0} \mathbf{x}} \\
& =\mathbf{I}+\frac{\partial^{t} \mathbf{u}^{\mathrm{e}}}{\partial^{\tau} \mathbf{x}^{\mathrm{p}}}+\frac{\partial^{t} \mathbf{u}^{\mathrm{p}}}{\partial^{0} \mathbf{x}}+\frac{\partial^{t} \mathbf{u}^{\mathrm{e}}}{\partial^{\tau} \mathbf{x}^{\mathrm{p}}} \frac{\partial^{t} \mathbf{u}^{\mathrm{p}}}{\partial^{0} \mathbf{x}}=\left(\mathbf{I}+\frac{\partial^{t} \mathbf{u}^{\mathrm{e}}}{\partial^{\tau} \mathbf{x}^{\mathrm{p}}}\right)\left(\mathbf{I}+\frac{\partial^{t} \mathbf{u}^{\mathrm{p}}}{\partial^{0} \mathbf{x}}\right)={ }_{\tau^{t}} \mathbf{X}^{\mathrm{e}}{ }_{0}^{\tau} \mathbf{X}^{\mathrm{p}}
\end{aligned}
$$

where ${ }_{\tau}^{t} \mathbf{X}^{\mathrm{e}}:=\partial^{t} \mathbf{X} / \partial^{\tau} \mathbf{x}^{\mathrm{p}}$ and ${ }_{0}^{\tau} \mathbf{X}^{\mathrm{p}}:=\partial^{\tau} \mathbf{x}^{\mathrm{p}} / \partial^{0} \mathbf{x}$ have the usual meaning and $\mathbf{I}$ is the second order identity tensor. Equation (5) may be interpreted also as a change of reference configuration from ${ }^{0} \mathbf{x}$ to ${ }^{\tau} \mathbf{x}^{\mathrm{p}}$-i.e. the configuration $\tau$ in the interpretation of Equation (3)-which is the view exploited in the numerical algorithm below. Obviously only ${ }^{0} \mathbf{x}$ and ${ }^{t} \mathbf{x}$ are tied to the kinematic compatibility constraint.

Of course, we could define ${ }^{t} \mathbf{x}^{\mathrm{e}}:={ }^{0} \mathbf{x}+{ }^{t} \mathbf{u}^{\mathrm{e}}$ as the co-ordinates of a dislocation-free configuration and obtain the reverse multiplicative decomposition, sometimes explored in the literature, see for example Reference [15] and references therein. However, since unloading occurs over the elastic part first, the decomposition (2) is far more natural and useful.

The spatial velocity gradient ${ }^{t} \mathbf{l}=\partial^{t} \mathbf{v} / \partial^{t} \mathbf{x}$ is

$$
{ }^{t} \mathbf{l}={ }_{0}^{t} \dot{\mathbf{X}}_{0}^{t} \mathbf{X}^{-1}
$$

and its symmetric part, the spatial deformation rate tensor, is

$$
{ }^{t} \mathbf{d}=\frac{1}{2}\left({ }_{0}^{t} \dot{\mathbf{X}}_{0}^{t} \mathbf{X}^{-1}+{ }_{0}^{t} \mathbf{X}^{-\mathrm{T} t}{ }_{0} \dot{\mathbf{X}}^{\mathrm{T}}\right)
$$

In view of Equation (2), Equation (6) can be decomposed as

$$
\begin{aligned}
\underbrace{\mathbf{l}} & =\mathbf{l}^{\mathrm{e}}+\mathbf{l}^{\mathrm{p}}=\underbrace{\mathbf{v}^{\mathrm{e}} / \partial^{t} \mathbf{x}}_{\partial^{t} \mathbf{v} / \partial^{t} \mathbf{x}}+\left(\partial^{t} \mathbf{x} / \partial^{\tau} \mathbf{x}^{\mathrm{p}}\right)\left[\partial^{\tau} \overline{\mathbf{v}}^{\mathrm{p}} / \partial^{\tau} \mathbf{x}^{\mathrm{p}}\right]\left(\partial^{\tau} \mathbf{x}^{\mathrm{p}} / \partial^{t} \mathbf{x}\right)
\end{aligned}
$$

where ${ }^{t} \mathbf{v}={ }^{t} \mathbf{v}^{\mathrm{e}}+\left(\partial^{t} \mathbf{x} / \partial^{\tau} \mathbf{x}^{\mathrm{p}}\right){ }^{\tau} \overline{\mathbf{v}}^{\mathrm{p}}$. The vector ${ }^{t} \mathbf{v}^{\mathrm{e}}\left({ }^{t} \mathbf{x}\right)={ }^{t} \dot{\mathbf{u}}^{\mathrm{e}}\left({ }^{t} \mathbf{x}\right)$ is the velocity with the plastic deformations frozen, ${ }^{\tau} \overline{\mathbf{v}}^{\mathrm{p}}\left({ }^{\tau} \mathbf{x}^{\mathrm{p}}\right)={ }^{\tau} \dot{\mathbf{u}}^{\mathrm{p}}\left({ }^{\tau} \mathbf{x}^{\mathrm{p}}\right)$ is the velocity with the elastic deformations and rigid body motions frozen and measured in the intermediate configuration, and ${ }^{t} \mathbf{v}^{\mathrm{p}}=$ $\left(\partial^{t} \mathbf{x} / \partial^{\tau} \mathbf{x}^{\mathrm{p}}\right){ }^{\tau} \overline{\mathbf{v}}^{\mathrm{p}}={ }^{t} \dot{\mathbf{u}}^{\mathrm{p}}\left({ }^{t} \mathbf{x}\right)$.

In the equations to follow in this section the time left-indices will be omitted since no confusion is possible given that all quantities are evaluated at time $t$. The tensor

$$
\overline{\mathbf{L}}^{\mathrm{p}}:=\dot{\mathbf{X}}^{\mathrm{p}}\left(\mathbf{X}^{\mathrm{p}}\right)^{-1}
$$

is the modified plastic velocity gradient, i.e. the velocity gradient in the intermediate configuration. The over-bar denotes that the tensor operates in the intermediate stress-free configuration. The symmetric part of $\overline{\mathbf{L}}^{\mathrm{p}}$ is the modified plastic deformation rate tensor

$$
\overline{\mathbf{D}}^{\mathrm{p}}:=\frac{1}{2}\left[\dot{\mathbf{X}}^{\mathrm{p}}\left(\mathbf{X}^{\mathrm{p}}\right)^{-1}+\left(\mathbf{X}^{\mathrm{p}}\right)^{-\mathrm{T}} \dot{\mathbf{X}}^{\mathrm{pT}}\right]
$$

while its skew part is the modified plastic spin

$$
\overline{\mathbf{W}}^{\mathrm{p}}:=\frac{1}{2}\left[\dot{\mathbf{X}}^{\mathrm{p}}\left(\mathbf{X}^{\mathrm{p}}\right)^{-1}-\left(\mathbf{X}^{\mathrm{p}}\right)^{-\mathrm{T}} \dot{\mathbf{X}}^{\mathrm{p} \mathrm{T}}\right]
$$


The tensor $\overline{\mathbf{D}}^{\mathrm{p}}$ is a consequence of the averaged Schmid rate of deformation tensors on all active slip mechanisms [15], while $\overline{\mathbf{W}}^{\mathrm{p}}$ is a consequence of the averaged Schmid rate of spin tensors on all active slip mechanisms which for isotropically oriented grains is commonly assumed to be zero. The pull-back of $\mathbf{l}^{\mathrm{e}}$ to the intermediate configuration is $\overline{\mathbf{L}}^{\mathrm{e}}:=\mathbf{X}^{\mathrm{eT}} \mathbf{l}^{\mathrm{e}} \mathbf{X}^{\mathrm{e}}=\mathbf{X}^{\mathrm{eT}} \dot{\mathbf{X}}^{\mathrm{e}}$, and the corresponding symmetric and skew parts are the modified elastic strain rate tensor and modified elastic spin tensor

$$
\overline{\mathbf{D}}^{\mathrm{e}}:=\frac{1}{2}\left(\mathbf{X}^{\mathrm{e} \mathrm{T}} \dot{\mathbf{X}}^{\mathrm{e}}+\dot{\mathbf{X}}^{\mathrm{eT}} \mathbf{X}^{\mathrm{e}}\right), \quad \overline{\mathbf{W}}^{\mathrm{e}}:=\frac{1}{2}\left(\mathbf{X}^{\mathrm{eT}} \dot{\mathbf{X}}^{\mathrm{e}}-\dot{\mathbf{X}}^{\mathrm{eT}} \mathbf{X}^{\mathrm{e}}\right)
$$

The symmetric part of $\mathbf{l}^{\mathrm{e}}$ is the spatial elastic deformation rate tensor $\mathbf{d}^{\mathrm{e}}=\frac{1}{2}\left[\dot{\mathbf{X}}^{\mathrm{e}}\left(\mathbf{X}^{\mathrm{e}}\right)^{-1}+\right.$ $\left.\left(\mathbf{X}^{\mathrm{e}}\right)^{-\mathrm{T}} \dot{\mathbf{X}}^{\mathrm{eT}}\right]$.

Some physical meaning of these symmetric tensors may be obtained from the Almansi strain tensors for each component of the deformation tensor defined in its proper configuration (the spatial configuration for the elastic strain tensor and the stress-free configuration for the plastic strain tensor):

$$
\mathbf{a}^{\mathrm{e}}=\frac{1}{2}\left[\mathbf{I}-\left(\mathbf{X}^{\mathrm{e}}\right)^{-\mathrm{T}}\left(\mathbf{X}^{\mathrm{e}}\right)^{-1}\right], \quad \overline{\mathbf{A}}^{\mathrm{p}}=\frac{1}{2}\left[\mathbf{I}-\left(\mathbf{X}^{\mathrm{p}}\right)^{-\mathrm{T}}\left(\mathbf{X}^{\mathrm{p}}\right)^{-1}\right]
$$

The corresponding Green-Lagrange strain tensors in their corresponding reference configurations are written as

$$
\overline{\mathbf{A}}^{\mathrm{e}}=\frac{1}{2}\left(\mathbf{X}^{\mathrm{eT}} \mathbf{X}^{\mathrm{e}}-\mathbf{I}\right), \quad \mathbf{A}^{\mathrm{p}}=\frac{1}{2}\left(\mathbf{X}^{\mathrm{pT}} \mathbf{X}^{\mathrm{p}}-\mathbf{I}\right)
$$

The time derivatives of these tensors with their reference configurations fixed are

$$
\dot{\overline{\mathbf{A}}}^{\mathrm{e}}=\frac{1}{2}\left(\dot{\mathbf{X}}^{\mathrm{e} \mathrm{T}} \mathbf{X}^{\mathrm{e}}+\mathbf{X}^{\mathrm{eT}} \dot{\mathbf{X}}^{\mathrm{e}}\right)=\overline{\mathbf{D}}^{\mathrm{e}}, \quad \dot{\mathbf{A}}^{\mathrm{p}}=\frac{1}{2}\left(\dot{\mathbf{X}}^{\mathrm{pT}} \mathbf{X}^{\mathrm{p}}+\mathbf{X}^{\mathrm{pT}} \dot{\mathbf{X}}^{\mathrm{p}}\right)
$$

and the covariant push-forward to the spatial and intermediate configurations gives the following quantities that may be viewed as Lie derivatives with $\mathbf{X}^{\mathrm{e}}$ and $\mathbf{X}^{\mathrm{p}}$ as gradients (i.e. relative to their reference configurations):

$$
\begin{gathered}
\mathscr{L}_{v}^{\mathrm{e}}\left(\mathbf{a}^{\mathrm{e}}\right)=\frac{1}{2}\left[\left(\mathbf{X}^{\mathrm{e}}\right)^{-\mathrm{T}} \dot{\mathbf{X}}^{\mathrm{eT}}+\dot{\mathbf{X}}^{\mathrm{e}}\left(\mathbf{X}^{\mathrm{e}}\right)^{-1}\right]=\mathbf{d}^{\mathrm{e}} \\
\mathscr{L}_{v}^{\mathrm{p}}\left(\overline{\mathbf{A}}^{\mathrm{p}}\right)=\frac{1}{2}\left[\left(\mathbf{X}^{\mathrm{p}}\right)^{-\mathrm{T}} \dot{\mathbf{X}}^{\mathrm{p} \mathrm{T}}+\dot{\mathbf{X}}^{\mathrm{p}}\left(\mathbf{X}^{\mathrm{p}}\right)^{-1}\right]=\overline{\mathbf{D}}^{\mathrm{p}}
\end{gathered}
$$

This view is exploited in the derivation of the consistent tangent moduli. If we define $\overline{\mathbf{L}}$, the modified velocity gradient as the pull-back of $\mathbf{l}$ to the intermediate configuration, we arrive at

$$
\overline{\mathbf{L}}=\overline{\mathbf{L}}^{\mathrm{e}}+\overline{\mathbf{C}}^{\mathrm{e}} \overline{\mathbf{L}}^{\mathrm{p}}
$$

where $\overline{\mathbf{C}}^{\mathrm{e}}=\mathbf{X}^{\mathrm{eT}} \mathbf{X}^{\mathrm{e}}$ is the right Cauchy-Green deformation tensor in the intermediate configuration. In passing we note that some of our definitions differ from those sometimes found in the literature. For example $\overline{\mathbf{D}}^{\mathrm{p}}$ is sometimes defined as $\overline{\mathbf{D}}^{\mathrm{p}}=\operatorname{sym}\left(\overline{\mathbf{C}}^{\mathrm{e}} \overline{\mathbf{L}}^{\mathrm{p}}\right)$, see for example 
References $[19,35,36]$. Nonetheless we believe that our definitions are more useful in the present work.

\subsection{Dissipation inequality}

The stress power in the reference volume may be expressed in the intermediate configuration as

$$
\begin{aligned}
\overline{\mathbf{S}}: \overline{\mathbf{L}} & =\overline{\mathbf{S}}:\left(\overline{\mathbf{L}}^{\mathrm{e}}+\mathbf{C}^{\mathrm{e}} \overline{\mathbf{L}}^{\mathrm{p}}\right) \\
& =\overline{\mathbf{S}}:\left(\overline{\mathbf{D}}^{\mathrm{e}}+\overline{\mathbf{W}}^{\mathrm{e}}\right)+\overline{\mathbf{S}}: \overline{\mathbf{C}}^{\mathrm{e}}\left(\overline{\mathbf{D}}^{\mathrm{p}}+\overline{\mathbf{W}}^{\mathrm{p}}\right)
\end{aligned}
$$

where $\overline{\mathbf{S}}$ is the pull-back of the Kirchhoff stress $\tau$ to the intermediate configuration. Since $\overline{\mathbf{S}}$ is symmetric the product $\overline{\mathbf{S}}: \overline{\mathbf{W}}^{\mathrm{e}}=0$, i.e. the modified elastic spin (which also contains the rigid-body spin) produces no work. Thus, we are left with

$$
\overline{\mathbf{S}}: \overline{\mathbf{L}}=\overline{\mathbf{S}}: \overline{\mathbf{D}}^{\mathrm{e}}+\overline{\mathbf{C}}^{\mathrm{e}} \overline{\mathbf{S}}:\left(\overline{\mathbf{D}}^{\mathrm{p}}+\overline{\mathbf{W}}^{\mathrm{p}}\right)
$$

where $\boldsymbol{\Xi}:=\overline{\mathbf{C}}^{\mathrm{e}} \overline{\mathbf{S}}$ is the non-symmetric Mandel stress tensor [37] forced by equilibrium of angular momentum to fulfil the condition $\overline{\mathbf{C}}^{\mathrm{e}-1} \boldsymbol{\Xi}=\boldsymbol{\Xi}^{\mathrm{T}} \overline{\mathbf{C}}^{\mathrm{e}-1}$. This tensor may be split into a symmetric part $\boldsymbol{\Xi}_{s}=\frac{1}{2}\left(\overline{\mathbf{C}}^{\mathrm{e}} \overline{\mathbf{S}}+\overline{\mathbf{S}} \overline{\mathbf{C}}^{\mathrm{e}}\right)$ and a skew one $\boldsymbol{\Xi}_{w}=\frac{1}{2}\left(\overline{\mathbf{C}}^{\mathrm{e}} \overline{\mathbf{S}}-\overline{\mathbf{S}} \overline{\mathbf{C}}^{\mathrm{e}}\right)$, yielding

$$
\begin{aligned}
\overline{\mathbf{S}}: \overline{\mathbf{L}} & =\overline{\mathbf{S}}: \overline{\mathbf{D}}^{\mathrm{e}}+\left(\boldsymbol{\Xi}_{s}+\boldsymbol{\Xi}_{w}\right):\left(\overline{\mathbf{D}}^{\mathrm{p}}+\overline{\mathbf{W}}^{\mathrm{p}}\right) \\
& =\overline{\mathbf{S}}: \overline{\mathbf{D}}^{\mathrm{e}}+\boldsymbol{\Xi}_{s}: \overline{\mathbf{D}}^{\mathrm{p}}+\boldsymbol{\Xi}_{w}: \overline{\mathbf{W}}^{\mathrm{p}}
\end{aligned}
$$

Thus, the symmetric Mandel tensor produces power on the modified plastic strain rate, whereas the skew-symmetric Mandel tensor produces power on the modified plastic spin. This last work is due to the kinematic coupling produced by the Lee decomposition and possible change of elastic anisotropy axes. In case of isotropy or deformation through the orthotropy axes, the term vanishes. Neglecting the effect of temperature, the dissipation inequality from the second law of thermodynamics is

$$
\begin{aligned}
\dot{\mathscr{D}} & =\tau: \mathbf{d}-\dot{\psi} \\
& =\overline{\mathbf{S}}: \overline{\mathbf{D}}^{\mathrm{e}}+\boldsymbol{\Xi}_{s}: \overline{\mathbf{D}}^{\mathrm{p}}+\boldsymbol{\Xi}_{w}: \overline{\mathbf{W}}^{\mathrm{p}}-\dot{\psi} \geqslant 0
\end{aligned}
$$

where $\psi$ is the free energy function. This free energy is a function of an elastic strain measure and other internal variables. In view of (15) we will express $\psi$ as a function of $\overline{\mathbf{A}}^{\mathrm{e}}$ and other strain-like set of internal variables $\{\bar{\xi}, \zeta\}$ (for simplicity, but without loss of generality, we consider in the present work that the set is formed by a tensor and a scalar). Thus

$$
\dot{\psi}=\frac{\partial \psi}{\partial \overline{\mathbf{A}}^{\mathrm{e}}}: \overline{\mathbf{D}}^{\mathrm{e}}+\frac{\partial \psi}{\partial \bar{\xi}}: \dot{\bar{\xi}}+\frac{\partial \psi}{\partial \zeta} \dot{\zeta}
$$

and

$$
\dot{\mathscr{D}}=\left(\overline{\mathbf{S}}-\frac{\partial \psi}{\partial \overline{\mathbf{A}}^{\mathrm{e}}}\right): \overline{\mathbf{D}}^{\mathrm{e}}-\frac{\partial \psi}{\partial \bar{\xi}}: \dot{\bar{\xi}}-\frac{\partial \psi}{\partial \zeta} \dot{\zeta}+\boldsymbol{\Xi}_{s}: \overline{\mathbf{D}}^{\mathrm{p}}+\boldsymbol{\Xi}_{w}: \overline{\mathbf{W}}^{\mathrm{p}} \geqslant 0
$$


Since the equality must hold for pure elastic deformations,

$$
\overline{\mathbf{S}}=\partial \psi / \partial \overline{\mathbf{A}}^{\mathrm{e}}
$$

By analogy we define a set of stress-like internal variables as $\overline{\boldsymbol{\beta}}=-\partial \psi / \partial \overline{\boldsymbol{\xi}}$ (backstress) and $\bar{\kappa}=-\partial \psi / \partial \zeta$ (overstress). The reduced (plastic) dissipation inequality is now

$$
\dot{\mathscr{D}}^{\mathrm{p}}=\overline{\boldsymbol{\beta}}: \dot{\bar{\xi}}+\bar{\kappa} \dot{\zeta}+\boldsymbol{\Xi}_{s}: \overline{\mathbf{D}}^{\mathrm{p}}+\boldsymbol{\Xi}_{w}: \overline{\mathbf{W}}^{\mathrm{p}} \geqslant 0
$$

If, as usual, we assume the existence of a yield function $f(\boldsymbol{\Xi}, \overline{\boldsymbol{\beta}}, \bar{\kappa})$ as the contour of allowed regions of stresses (for simplicity but, without loss of generality, we assume in the present work that the contour may be expressed in terms of a single function $f$ ), the Lagrangian for the constrained problem is $L=\dot{\mathscr{D}}^{\mathrm{p}}-\dot{t} f$, were $t$ is the consistency parameter. If we claim that the principle of maximum dissipation holds, the stress and other internal variables are such that $\nabla L=0$, i.e.

$$
\nabla L=0 \Rightarrow\left\{\begin{array}{l}
\frac{\partial L}{\partial \mathbf{\Xi}_{s}}=0 \Rightarrow \overline{\mathbf{D}}^{\mathrm{p}}=\dot{t} \frac{\partial f}{\partial \mathbf{\Xi}_{s}} \\
\frac{\partial L}{\partial \mathbf{\Xi}_{w}}=0 \Rightarrow \overline{\mathbf{W}}^{\mathrm{p}}=\dot{t} \frac{\partial f}{\partial \mathbf{\Xi}_{w}} \\
\frac{\partial L}{\partial \overline{\boldsymbol{\beta}}}=0 \Rightarrow \dot{\bar{\xi}}=\dot{t} \frac{\partial f}{\partial \overline{\boldsymbol{\beta}}} \\
\frac{\partial L}{\partial \bar{\kappa}}=0 \Rightarrow \dot{\zeta}=\dot{t} \frac{\partial f}{\partial \bar{\kappa}}
\end{array}\right.
$$

These expressions are the associated flow and hardening rules for general elasto-plasticity at finite strains.

\subsection{Isotropic elasticity and the logarithmic stored energy function}

We consider in the rest of this paper the special case when the elastic part is isotropic. In this case, the eigenvectors of $\overline{\mathbf{S}}, \overline{\mathbf{A}}^{\mathrm{e}}$ and $\overline{\mathbf{C}}^{\mathrm{e}}$ are coincident, the matrices commute, and therefore $\boldsymbol{\Xi}_{w}=\mathbf{0}$. The important consequence is that the plastic spin does not dissipate energy in isotropic elasticity and that $\boldsymbol{\Xi}_{s}$ alone fulfils the equations of angular momenta. Otherwise, in general a change in $\boldsymbol{\Xi}_{w}$ is needed to re-establish the equilibrium of angular momentum lost by the local symmetric plastic flow.

On the other hand, due to the commutative property, using the right polar decomposition for the elastic deformation tensor $\mathbf{X}^{\mathrm{e}}=\mathbf{R}^{\mathrm{e}} \mathbf{U}^{\mathrm{e}}$, the symmetric Mandel tensor may be written as

$$
\boldsymbol{\Xi}_{s}=\frac{1}{2}\left(\overline{\mathbf{C}}^{\mathrm{e}} \overline{\mathbf{S}}+\overline{\mathbf{S}} \overline{\mathbf{C}}^{\mathrm{e}}\right)=\mathbf{U}^{\mathrm{e}} \overline{\mathbf{S}} \mathbf{U}^{\mathrm{e}}
$$

which in terms of the spatial Kirchhoff stress tensor may be written as

$$
\boldsymbol{\Xi}=\boldsymbol{\Xi}_{s}=\mathbf{U}^{\mathrm{e}} \mathbf{X}^{\mathrm{e}-1} \tau \mathbf{X}^{\mathrm{e}-\mathrm{T}} \mathbf{U}^{\mathrm{e}}=\mathbf{R}^{\mathrm{e} \mathrm{T}} \tau \mathbf{R}^{\mathrm{e}}=\bar{\tau}
$$

i.e. in isotropic elasticity the Mandel stress tensor coincides with the usually named rotated Kirchhoff stress tensor $\bar{\tau}$, see for example References [21,32,33]. 
One of the major difficulties encountered in the development of algorithms was due to the multiplicative nature of large strain elasto-plasticity. Weber and Anand [20] and Eterović and Bathe [21] circumvented the problem using hyperelastic stored energy functions based on logarithmic strain measures. As mentioned, this function has been shown to correlate well with experiments in the range of moderately large elastic strains, see Reference [30]. Denoting by $\lambda_{i}$ the principal elastic stretches, by $J=\operatorname{det}(\mathbf{X})$ the Jacobian determinant and by $\hat{\lambda}_{i}=J^{-1 / 3} \lambda_{i}$ the isochoric principal elastic stretches, we have:

$$
\mathscr{W}\left(\lambda_{1}, \lambda_{2}, \lambda_{3}\right)=\mathscr{U}(J)+\mu \sum_{i=1}^{3}\left(\ln \hat{\lambda}_{i}\right)^{2}
$$

where $\mu$ is interpreted as the shear modulus and $\mathscr{U}(J)$ is the volumetric contribution. Applying the chain rule to Equation (25) in principal directions we obtain (no sum on $i$ ):

$$
\bar{S}_{i}=\sum_{j=1}^{3} \frac{\partial \mathscr{W}}{\partial \ln \lambda_{j}} \frac{\partial \ln \lambda_{j}}{\partial\left(\frac{1}{2} \lambda_{i}^{2}\right)}=\sum_{j=1}^{3} \frac{\partial \mathscr{W}}{\partial \ln \lambda_{j}} \frac{\delta_{i j}}{\lambda_{i} \lambda_{j}}=\frac{1}{\lambda_{i}^{2}} \frac{\partial \mathscr{W}}{\partial \ln \lambda_{i}}
$$

Taking into account that

$$
\sum_{i=1}^{3} \ln \hat{\lambda}_{i}=0, \quad \frac{\partial \hat{\lambda}_{i}}{\partial \lambda_{j}}=J^{-1 / 3}\left(\delta_{i j}-\frac{1}{3} \frac{\hat{\lambda}_{i}}{\hat{\lambda}_{j}}\right)
$$

from (30),

$$
\begin{aligned}
\frac{\partial \mathscr{W}}{\partial \ln \lambda_{i}} & =\sum_{k=1}^{3} \mathscr{U}^{\prime}(J) \frac{\partial J}{\partial \lambda_{k}} \frac{\partial \lambda_{k}}{\partial \ln \lambda_{i}}+2 \mu \sum_{k=1}^{3} \frac{1}{\hat{\lambda}_{k}}\left(\ln \hat{\lambda}_{k}\right) \frac{\partial \hat{\lambda}_{k}}{\partial \lambda_{i}} \frac{\partial \lambda_{i}}{\partial \ln \lambda_{i}} \\
& =\mathscr{U}^{\prime}(J) J+2 \mu \ln \hat{\lambda}_{i}
\end{aligned}
$$

Using (28) and (31), the symmetric Mandel stress tensor for this isotropic stored energy function results in

$$
\boldsymbol{\Xi} \equiv \boldsymbol{\Xi}_{s} \equiv \bar{\tau}=\frac{\partial \mathscr{W}}{\partial \mathbf{E}^{\mathrm{e}}}=J \mathscr{U}^{\prime}(J) \mathbf{I}+2 \mu \mathbf{E}^{\mathrm{ed}}
$$

where $\mathbf{E}^{\mathrm{ed}}=\ln \left(J^{-1 / 3} \mathbf{U}^{\mathrm{e}}\right)$ and $\mathbf{E}^{\mathrm{e}}=\ln \mathbf{U}^{\mathrm{e}}=\frac{1}{3}(\ln J) \mathbf{I}+\mathbf{E}^{\mathrm{ed}}$ are the Hencky deviatoric and total strain tensors. The proportionality relationship between $\bar{\tau}$ and $\mathbf{E}^{\mathrm{e}}$ is one of the main ingredients in the development of the additive plastic return algorithm. Thus, for non-deviatoric plasticity $J \mathscr{U}^{\prime}(J)$ must also be linear in $\ln J$. For deviatoric plasticity other functions $\mathscr{U}(J)$ may still be used but, as shown below, some modifications are necessary. Stored energy functions $\mathscr{W}$ that result in stresses non-linear in the logarithmic strains, different from (30), may still be used, but from a numerical point of view they may require an additional iterative process in the stress integration algorithm.

In passing we note that $\overline{\mathbf{S}}: \overline{\mathbf{D}}^{\mathrm{e}}=\boldsymbol{\Xi}: \overline{\mathbf{C}}^{\mathrm{e}-1} \overline{\mathbf{D}}^{\mathrm{e}}$. In the case of isotropic elasticity $\boldsymbol{\Xi}=\bar{\tau}$ and by straightforward use of the spectral decomposition, taking into account that the eigenvectors of $\bar{\tau}$ coincide with those of $\overline{\mathbf{C}}^{\mathrm{e}}$, it can be shown that $\bar{\tau}: \overline{\mathbf{C}}^{\mathrm{e}-1} \overline{\mathbf{D}}^{\mathrm{e}}=\bar{\tau}: \dot{\mathbf{E}}^{\mathrm{e}}=\sum_{i=1}^{3} \tau_{i} \dot{\lambda}_{i} / \lambda_{i}$ (with $\tau_{i}$ the principal spatial Kirchhoff stresses). Thus, we are left with the stress power and 
dissipation expressions of [21]:

$$
\dot{\mathscr{D}}=\bar{\tau}: \dot{\mathbf{E}}^{\mathrm{e}}+\bar{\tau}: \overline{\mathbf{D}}^{\mathrm{p}}-\dot{\psi} \geqslant 0
$$

Nonetheless this form of the dissipation inequality is not necessary for the development of the algorithm since it is implicitly employed by Equation (34) through the use of the chain rule.

\subsection{The spatial and principal stress space formulations}

The large strain elastoplasticity based on isotropic elastic response and logarithmic stretches has also been developed in the spatial configuration, see for example Reference [22]. Of course, both formulations result into identical response predictions. Using the left polar decomposition theorem $\mathbf{X}^{\mathrm{e}}=\mathbf{V}^{\mathrm{e}} \mathbf{R}^{\mathrm{e}}$, we define $\varepsilon^{\mathrm{e}}=\ln \mathbf{V}^{\mathrm{e}}=\mathbf{R}^{\mathrm{e}} \mathbf{E}^{\mathrm{e}} \mathbf{R}^{\mathrm{eT}}$. Since $\tau=\mathbf{R}^{\mathrm{e}} \bar{\tau} \mathbf{R}^{\mathrm{eT}}=J \mathscr{U}^{\prime}(J) \mathbf{I}+2 \mu \boldsymbol{\varepsilon}^{\mathrm{ed}}$ we have from (34)

$$
\tau=\frac{\partial \mathscr{W}}{\partial \boldsymbol{\varepsilon}^{\mathrm{e}}}=2 \frac{\partial \mathscr{W}}{\partial \mathbf{b}^{\mathrm{e}}} \mathbf{b}^{\mathrm{e}}
$$

where $\mathbf{b}^{\mathrm{e}}$ is the elastic Finger tensor and $\boldsymbol{\varepsilon}^{\mathrm{ed}}$ is the deviatoric part of $\boldsymbol{\varepsilon}^{\mathrm{e}}$. The second identity in (36) is obtained using the spectral decomposition. Also, using the symmetry of $\bar{\tau}$, the isotropy of elastic response yields the following identities:

$$
\bar{\tau}: \overline{\mathbf{D}}^{\mathrm{p}}=\mathbf{U}^{\mathrm{e}-1} \bar{\tau} \mathbf{U}^{\mathrm{e}}: \overline{\mathbf{L}}^{\mathrm{p}}=\mathbf{R}^{\mathrm{e} \mathrm{T}} \tau \mathbf{R}^{\mathrm{e}}: \mathbf{U}^{\mathrm{e}} \overline{\mathbf{L}}^{\mathrm{p}} \mathbf{U}^{\mathrm{e}-1}=\tau: \mathbf{X}^{\mathrm{e}} \overline{\mathbf{L}}^{\mathrm{p}} \mathbf{X}^{\mathrm{e}-1}=\tau: \mathbf{l}^{\mathrm{p}}=\tau: \mathbf{d}^{\mathrm{p}}
$$

These expressions inserted in the dissipation inequality give for (27) the spatial formulation encountered, for example, in Reference [22]. In fact, given an arbitrary rotation $\mathbf{Q}$ on the intermediate (rotated) configuration, the following tensors can be defined:

$$
\tilde{\tau}:=\mathbf{Q}^{\mathrm{T}} \bar{\tau} \mathbf{Q}, \quad \tilde{\mathbf{d}}^{\mathrm{p}}=\mathbf{Q}^{\mathrm{T}} \overline{\mathbf{D}}^{\mathrm{p}} \mathbf{Q}, \quad \tilde{\boldsymbol{\varepsilon}}^{\mathrm{e}}=\mathbf{Q}^{\mathrm{T}} \ln \mathbf{U}^{\mathrm{e}} \mathbf{Q}
$$

and still $\tilde{\tau}=\partial \mathscr{W} / \partial \tilde{\boldsymbol{\varepsilon}}^{\mathrm{e}}$, and $\tau: \mathbf{d}^{\mathrm{p}}=\tilde{\tau}: \tilde{\mathbf{d}}^{\mathrm{p}}$. This property was used for the development of a stress integration algorithm in the principal stress space, considering isotropic plasticity in Reference [22] (simply set $\mathbf{Q}=\sum_{i=1}^{3} \mathbf{N}_{i} \otimes \mathbf{e}_{i}$, where $\mathbf{N}_{i}$ are the eigenvectors of $\bar{\tau}$ and $\mathbf{e}_{i}$ are the fixed Cartesian unit vectors). However, as shown below, the integration in a reduced dimension of (three) principal stresses produces different results in the case of kinematic/combined hardening due to the back-stress evolution.

\section{NUMERICAL ALGORITHM INCLUDING PLASTIC SPIN}

In this section we use the time super- and subscripts to clearly indicate the time integration used.

\subsection{General formulation}

From (9) the evolution of the plastic deformation gradient tensor is given by the differential equation

$$
{ }_{0}^{t} \dot{\mathbf{X}}^{\mathrm{p}}={ }^{t} \overline{\mathbf{L}}^{\mathrm{p}}{ }_{0}^{t} \mathbf{X}^{\mathrm{p}}
$$


whose backward-Euler exponential solution is given by

$$
{ }_{0}^{t+\Delta t} \mathbf{X}^{\mathrm{p}}=\exp \left(\Delta t{ }^{t+\Delta t} \overline{\mathbf{L}}^{\mathrm{p}}\right){ }_{0}^{t} \mathbf{X}^{\mathrm{p}}
$$

where the exponential function of a matrix $\exp \left(\Delta t^{t+\Delta t} \overline{\mathbf{L}}^{\mathrm{p}}\right)$ is defined as

$$
\exp \left(\Delta t^{t+\Delta t} \overline{\mathbf{L}}^{\mathrm{p}}\right):=\sum_{n=0}^{\infty} \frac{\left(\Delta t^{t+\Delta t} \overline{\mathbf{L}}^{\mathrm{p}}\right)^{n}}{n !}
$$

and for small steps such that $h:=\left\|\Delta t^{t+\Delta t} \overline{\mathbf{L}}^{\mathrm{p}}\right\| \ll 1$ can be approximated by

$$
\exp \left(\Delta t^{t+\Delta t} \overline{\mathbf{L}}^{\mathrm{p}}\right)=\mathbf{I}+\Delta t^{t+\Delta t} \overline{\mathbf{L}}^{\mathrm{p}}+\cdots+\mathcal{O}\left(h^{2}\right)
$$

Also, since ${ }^{t+\Delta t} \overline{\mathbf{L}}^{\mathrm{p}}={ }^{t+\Delta t} \overline{\mathbf{D}}^{\mathrm{p}}+{ }^{t+\Delta t} \overline{\mathbf{W}}^{\mathrm{p}}$, we thus have

$$
\exp \left(\Delta t{ }^{t+\Delta t} \overline{\mathbf{L}}^{\mathrm{p}}\right)=\exp \left(\Delta t{ }^{t+\Delta t} \overline{\mathbf{D}}^{\mathrm{p}}\right) \exp \left(\Delta t{ }^{t+\Delta t} \overline{\mathbf{W}}^{\mathrm{p}}\right)+\cdots+\mathcal{O}\left(h^{2}\right)
$$

yielding the following useful update formulas:

$$
{ }_{0}^{t+\Delta t} \mathbf{X}^{\mathrm{p}-1}={ }_{0}^{t} \mathbf{X}^{\mathrm{p}-1} \exp \left(-\Delta t{ }^{t+\Delta t} \overline{\mathbf{W}}^{\mathrm{p}}\right) \exp \left(-\Delta t{ }^{t+\Delta t} \overline{\mathbf{D}}^{\mathrm{p}}\right)
$$

and

$$
{ }_{0}^{t+\Delta t} \mathbf{X}^{\mathrm{e}}=\mathbf{X}_{*}^{\mathrm{e}} \exp \left(-\Delta t{ }^{t+\Delta t} \overline{\mathbf{W}}^{\mathrm{p}}\right) \exp \left(-\Delta t^{t+\Delta t} \overline{\mathbf{D}}^{\mathrm{p}}\right)
$$

where the tensor $\mathbf{X}_{*}^{\mathrm{e}} \equiv{ }^{t+\Delta t} \mathbf{X}_{*}^{\mathrm{e}}:={ }^{t+\Delta t}{ }_{t} \mathbf{X}_{0}^{t} \mathbf{X}^{\mathrm{e}}$ is the trial elastic deformation gradient (i.e. with the plastic state frozen). For trial tensors we omit the left time superindex since they are always evaluated at $t+\Delta t$ and no confusion is possible. In the present work we have used for simplicity the same consistency parameter $\Delta t$ for both the symmetric and the skew parts. However, ${ }^{t+\Delta t} \overline{\mathbf{D}}^{\mathrm{p}}$ and ${ }^{t+\Delta t} \overline{\mathbf{W}}^{\mathrm{p}}$ are frequently defined up to a scalar parameter and sometimes different consistency parameters may be necessary. The polar decomposition theorem yields

$$
\mathbf{X}_{*}^{\mathrm{e}}=\mathbf{R}_{*}^{\mathrm{e}} \mathbf{U}_{*}^{\mathrm{e}}
$$

where $\mathbf{U}_{*}^{\mathrm{e}}$ is the trial (elastic) right stretch tensor and $\mathbf{R}_{*}^{\mathrm{e}}$ is the trial rotation tensor.

Now defining

$$
{ }^{t+\Delta t}{ }_{t} \mathbf{R}^{w}:=\exp \left(\Delta t{ }^{t+\Delta t} \overline{\mathbf{W}}^{\mathrm{p}}\right)
$$

which is an orthogonal tensor and using (45) and $\mathbf{C}_{*}^{\mathrm{e}}:=\mathbf{X}_{*}^{\mathrm{eT}} \mathbf{X}_{*}^{\mathrm{e}}$ (the trial right Cauchy-Green deformation tensor) we obtain

$$
\begin{aligned}
\mathbf{C}_{*}^{\mathrm{e}}= & { }^{t+\Delta t}{ }_{t} \mathbf{R}^{w \mathrm{~T}} \exp \left(\Delta t{ }^{t+\Delta t} \overline{\mathbf{D}}^{\mathrm{p}}\right){ }_{0}^{t+\Delta t} \mathbf{C}^{\mathrm{e}} \exp \left(\Delta t{ }^{t+\Delta t} \overline{\mathbf{D}}^{\mathrm{p}}\right){ }^{t+\Delta t}{ }_{t}^{t+} \mathbf{R}^{w} \\
= & {\left[{ }^{t+\Delta t}{ }_{t} \mathbf{R}^{w \mathrm{~T}} \exp \left(\Delta t{ }^{t+\Delta t} \overline{\mathbf{D}}^{\mathrm{p}}\right){ }^{t+\Delta t}{ }_{t} \mathbf{R}^{w}\right]\left[{ }^{t+\Delta t}{ }_{t} \mathbf{R}^{w \mathrm{~T} t+\Delta t} \mathbf{C}^{\mathrm{e} t+\Delta t}{ }_{t} \mathbf{R}^{w}\right] } \\
& \times\left[{ }^{t+\Delta t}{ }_{t} \mathbf{R}^{w \mathrm{~T}} \exp \left(\Delta t{ }^{t+\Delta t} \overline{\mathbf{D}}^{\mathrm{p}}\right){ }^{t+\Delta t}{ }_{t} \mathbf{R}^{w}\right]
\end{aligned}
$$


The following tensors are next defined

$$
\begin{gathered}
{ }^{t+\Delta t} \tilde{\mathbf{C}}^{\mathrm{e}}:={ }^{t+\Delta t}{ }_{t} \mathbf{R}^{w \mathrm{~T} t+\Delta t}{ }_{0} \mathbf{C}^{\mathrm{e} t+\Delta t}{ }_{t} \mathbf{R}^{w} \\
\exp \left(\Delta t{ }^{t+\Delta t} \tilde{\mathbf{D}}^{\mathrm{p}}\right):={ }^{t+\Delta t}{ }_{t} \mathbf{R}^{w \mathrm{~T}} \exp \left(\Delta t{ }^{t+\Delta t} \overline{\mathbf{D}}^{\mathrm{p}}\right){ }^{t+\Delta t} \mathbf{R}^{w}
\end{gathered}
$$

where ${ }_{0}^{t+\Delta t} \mathbf{C}^{\mathrm{e}}:=\left({ }_{0}^{t+\Delta t} \mathbf{X}^{\mathrm{e}}\right)^{\mathrm{T}}{ }_{0}^{t+\Delta t} \mathbf{X}^{\mathrm{e}}$ and is, of course, unknown. We also define $\mathbf{E}_{*}^{\mathrm{e}}$ (the trial Hencky strain tensor) and ${ }^{t+\Delta t} \tilde{\mathbf{E}}^{\mathrm{e}}$ such that $\mathbf{C}_{*}^{\mathrm{e}}=: \exp \left(2 \mathbf{E}_{*}^{\mathrm{e}}\right)$ and ${ }_{0}^{t+\Delta t} \tilde{\mathbf{C}}^{\mathrm{e}}=: \exp \left(2_{0}^{t+\Delta t} \tilde{\mathbf{E}}^{\mathrm{e}}\right)$. Using (42), Equation (49) may be re-written as

$$
\mathbf{E}_{*}^{\mathrm{e}} \simeq{ }_{0}^{t+\Delta t} \tilde{\mathbf{E}}^{\mathrm{e}}+\Delta t^{t+\Delta t} \tilde{\mathbf{D}}^{\mathrm{p}}
$$

with the additional restriction that $h^{*}:=\left\|\mathbf{E}_{*}^{\mathrm{e}}\right\| \ll 1$, i.e. the elastic strains and incremental steps are only moderately large, which typically holds in metal plasticity. Note that the restriction $h^{*} \ll 1$ is more restrictive than $h \ll 1$. Defining the trial unrotated stress and backstress tensors

$$
\overline{\mathbf{T}}_{*}:=\bar{\tau}_{*}:=\frac{\partial \mathscr{W}}{\partial \mathbf{E}_{*}^{\mathrm{e}}} \quad \text { and } \quad \overline{\mathbf{B}}_{*}:={ }^{t} \overline{\boldsymbol{\beta}}
$$

from $\left(27_{1}\right)$ and (51) we have

$$
\overline{\mathbf{D}}_{*}^{\mathrm{p}}=\frac{\partial f\left(\overline{\mathbf{T}}_{*}-\overline{\mathbf{B}}_{*},{ }^{t} \bar{\kappa}\right)}{\partial \overline{\mathbf{T}}_{*}}
$$

and for the case of $J_{2}$ plasticity ${ }^{t+\Delta t} \tilde{\mathbf{D}}^{\mathrm{p}}=\overline{\mathbf{D}}_{*}^{\mathrm{p}}$. Equation (52) is recognized as the incrementally additive decomposition of logarithmic strains that allows for the use of the small strain algorithms to perform the plastic return. In this plastic return the tensor ${ }_{0}^{t+\Delta t} \tilde{\mathbf{E}}^{\mathrm{e}}$ in (52) —or more accurately the tensor ${ }^{t+\Delta t} \overline{\mathbf{T}}$-is obtained. If the plastic spin is non-zero, the resulting strain (stress) is a rotated one. The correct stress is then obtained using (50) and the integration of any proper constitutive equation for the plastic spin. Which constitutive relation to use is in general an open issue.

Taking the volumetric and deviatoric parts of (52) for the particular case of deviatoric plasticity, the stress update is performed through the set of equations

$$
\begin{aligned}
\bar{\tau}^{\mathrm{vol}} & =\bar{\tau}^{\mathrm{vol}}(\ln J) \\
{ }^{t+\Delta t} \overline{\mathbf{T}}^{\mathrm{d}} & =2 \mu{ }_{0}^{t+\Delta t} \tilde{\mathbf{E}}^{\mathrm{ed}}, \quad{ }^{t+\Delta t} \bar{\tau}={ }_{t}^{t+\Delta t}{ }_{t} \mathbf{R}^{w t+\Delta t} \overline{\mathbf{T}}^{t+\Delta t}{ }_{t} \mathbf{R}^{w \mathrm{~T}} \\
{ }^{t+\Delta t} \overline{\boldsymbol{\beta}} & ={ }^{t+\Delta t}{ }_{t} \mathbf{R}^{w}{ }^{t+\Delta t} \overline{\mathbf{B}}^{t+\Delta t}{ }_{t} \mathbf{R}^{w \mathrm{~T}}
\end{aligned}
$$

where $(\cdot)^{\mathrm{d}}$ denotes the deviatoric part. Note that the plastic spin is skew and produces no work on symmetric tensors, hence the plastic spin is decoupled from the symmetric plastic return, unless the specific constitutive equation employed for the plastic spin couples them. In such a case a simultaneous integration should be performed. The spatial Kirchhoff stress and backstress are finally obtained as

$$
{ }^{t+\Delta t} \tau=\mathbf{R}_{*}^{\mathrm{e} t+\Delta t} \bar{\tau} \mathbf{R}_{*}^{\mathrm{eT}}, \quad{ }^{t+\Delta t} \boldsymbol{\beta}=\mathbf{R}_{*}^{\mathrm{e} t+\Delta t} \overline{\boldsymbol{\beta}} \mathbf{R}_{*}^{\mathrm{eT}}
$$


The integration may also be performed in any arbitrarily rotated configuration instead of that given by $\mathbf{Q}=\mathbf{R}_{*}^{\mathrm{eT}}$, which is an obvious consequence of the objectivity principle that must hold even for the integration algorithm. We note that the use of our choice makes unnecessary the update of the backstress by $\left(58_{2}\right)$ since this rotated configuration is invariant to rigid body motions.

The rotation component of the trial elastic gradient tensor is obtained by use of the right polar decomposition theorem and formulas (45) and (42) as:

$$
\begin{aligned}
\mathbf{R}_{*}^{\mathrm{e}}= & { }_{0}^{t+\Delta t} \mathbf{R}^{\mathrm{e}} \exp \left({ }^{t+\Delta t} \mathbf{E}^{\mathrm{e}}\right) \exp \left(\Delta t{ }^{t+\Delta t} \overline{\mathbf{D}}^{\mathrm{p}}\right) \exp \left(\Delta t{ }^{t+\Delta t} \overline{\mathbf{W}}^{\mathrm{p}}\right) \exp \left(-\mathbf{E}_{*}^{\mathrm{e}}\right) \\
= & { }^{t+\Delta t}{ }_{0} \mathbf{R}^{\mathrm{e} t+\Delta t}{ }_{t} \mathbf{R}^{w} \exp \left({ }^{t+\Delta t} \tilde{\mathbf{E}}^{\mathrm{e}}\right){ }^{t+\Delta t}{ }_{t} \mathbf{R}^{w \mathrm{~T}} \\
& \times{ }^{t+\Delta t}{ }_{t} \mathbf{R}^{w} \exp \left(\Delta t{ }^{t+\Delta t} \tilde{\mathbf{D}}^{\mathrm{p}}\right){ }^{t+\Delta t} \mathbf{R}_{t}{ }^{w \mathrm{~T}}{ }^{t+\Delta t}{ }_{t} \mathbf{R}^{w} \exp \left(-\mathbf{E}_{*}^{\mathrm{e}}\right) \\
= & { }^{t+\Delta t}{ }_{0} \mathbf{R}^{\mathrm{e} t+\Delta t}{ }_{t} \mathbf{R}^{w}+\cdots+\mathcal{O}\left(h^{*}\right)^{2}
\end{aligned}
$$

which yields

$$
{ }_{0}^{t+\Delta t} \mathbf{R}^{\mathrm{e}} \simeq \mathbf{R}_{*}^{\mathrm{e} t+\Delta t}{ }_{t} \mathbf{R}^{w \mathrm{~T}}
$$

and so we note that the elastic part of the rotation ${ }_{0}^{t+\Delta t} \mathbf{R}^{\mathrm{e}}$ changes with the incremental plastic rotation ${ }^{t+\Delta t}{ }_{t} \mathbf{R}^{w}$. The main tensors and configurations used in the stress integration algorithm are depicted in Figure 1, whereas the layout of the stress integration algorithm is given in Table I.

Equation (52) constitutes the basis for the algorithm developed in this paper. However, depending on the constitutive equation for the plastic spin, a different form may be sometimes

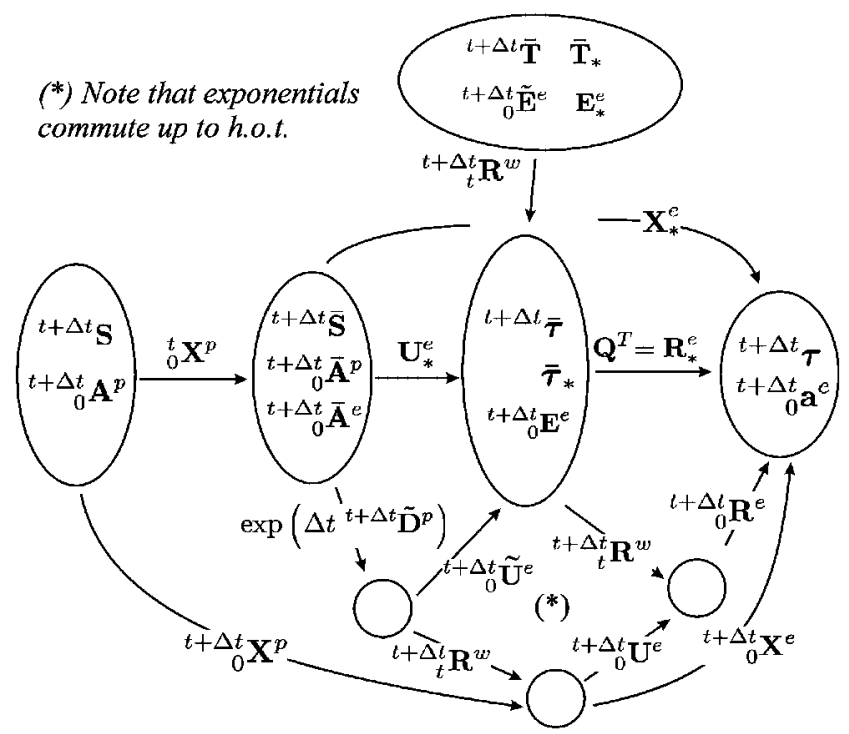

Figure 1. Configurations and tensors in the stress integration algorithm. 
Table I. Stress integration algorithm for the total Lagrangian (TL) and updated Lagrangian (UL) formulations.

Given ${ }^{t} \overline{\boldsymbol{\beta}},{ }^{t} \bar{\kappa}$, and $\left({ }_{0}^{t} \mathbf{X}^{\mathrm{p}-1}\right.$ and ${ }^{t+\Delta t} \mathbf{X}$ in TL) or $\left({ }_{0}^{t} \mathbf{X}^{\mathrm{e}}\right.$ and ${ }^{t+\Delta t} \mathbf{X}$ in UL)

1. Obtain trial elastic tensor: $\mathbf{X}_{*}^{\mathrm{e}}={ }^{t+\Delta t}{ }_{0} \mathbf{X}_{0}^{t} \mathbf{X}^{\mathrm{p}-1}={ }^{t+\Delta t}{ }_{t}{ }_{0}^{t} \mathbf{X}^{\mathrm{e}}$

2. Compute trial Cauchy-Green deformation tensor $\mathbf{C}_{*}^{\mathrm{e}}=\mathbf{X}_{*}^{\mathrm{eT}} \mathbf{X}_{*}^{\mathrm{e}}$

3. Obtain $\lambda_{i}^{*}, \mathbf{N}_{i}^{*} \otimes \mathbf{N}_{i}^{*}$ and $\mathbf{U}_{*}^{\mathrm{e}}=\sum_{i=1}^{3} \lambda_{i}^{*} \mathbf{N}_{i}^{*} \otimes \mathbf{N}_{i}^{*}$ and $\mathbf{R}_{*}^{\mathrm{e}}=\mathbf{X}_{*}^{\mathrm{e}} \mathbf{U}_{*}^{\mathrm{e}-1}\left(J=\operatorname{det}{ }^{t+\Delta t} \mathbf{X}>0\right)$

4. Compute rotated trial stress $\bar{\tau}_{*}=\overline{\mathbf{T}}_{*}=J \mathscr{U}^{\prime}(J) \mathbf{I}+2 \mu \sum_{i=1}^{3} \ln \left(J^{-1 / 3} \lambda_{i}^{*}\right) \mathbf{N}_{i}^{*} \otimes \mathbf{N}_{i}^{*}$ and set $\overline{\mathbf{B}}_{*}={ }^{t} \overline{\boldsymbol{\beta}}$; $\bar{\kappa}_{*}={ }^{t} \bar{\kappa}$

$\int$ Call small strains subroutine to perform stress integration returning : 5. $\left\{\begin{array}{l}{ }_{t+\Delta t} \overline{\mathbf{T}} \text { (as stress), }{ }^{t+\Delta t} \overline{\mathbf{B}} \text { (backstress), } \Delta t{ }^{t+\Delta t} \tilde{\mathbf{D}}^{\mathrm{p}} \text { (plastic strain incr.), }{ }^{t+\Delta t} \bar{\kappa} \\ { }_{t+\Delta t} \tilde{\mathbb{D}} \text { (as constitutive tensor) }\end{array}\right.$

6. Integrate plastic spin obtaining ${ }^{t+\Delta t}{ }_{t} \mathbf{R}^{w}:=\exp \left(\Delta t{ }^{t+\Delta t} \overline{\mathbf{W}}^{\mathrm{p}}\right)$

7. Rotate $\left\{{ }^{t+\Delta t} \bar{\tau},{ }^{t+\Delta t} \overline{\boldsymbol{\beta}}\right\}={ }^{t+\Delta t}{ }_{t} \mathbf{R}^{w}\left\{{ }^{t+\Delta t} \overline{\mathbf{T}},{ }^{t+\Delta t} \overline{\mathbf{B}}\right\}{ }^{t+\Delta t}{ }_{t} \mathbf{R}^{w T}$

8. Obtain Cauchy stress $J^{-1 t+\Delta t} \tau=J^{-1} \mathbf{R}_{*}^{\mathrm{e} t+\Delta t} \bar{\tau} \mathbf{R}_{*}^{\mathrm{eT}}{ }^{t}$

9. During iterative phase compute consistent tangent ${ }^{t+\Delta t} \mathbb{C}^{(i)}$ as shown in Section 4 (correct the volumetric part of ${ }^{t+\Delta t} \tilde{\mathbb{D}}$ if necessary and push/pull ${ }^{t+\Delta t} \mathbb{C}^{(i)}$ as needed)

10. In convergence phase update ${ }^{t+\Delta t} \mathbf{X}^{\mathrm{p}-1}={ }_{0}^{t} \mathbf{X}^{\mathrm{p}-1} \exp \left(-\Delta t{ }^{t+\Delta t} \tilde{\mathbf{D}}^{\mathrm{p}}\right)^{t+\Delta t} \mathbf{R}^{w \mathrm{~T}}$ if $\mathrm{TL}$ or ${ }^{t+\Delta t} \mathbf{X}^{\mathrm{e}}=$ $\mathbf{X}_{*}^{\mathrm{e}} \exp \left(-\Delta t{ }^{t+\Delta t} \tilde{\mathbf{D}}^{\mathrm{p}}\right)^{t+\Delta t} \mathbf{R}^{w \mathrm{~T}}$ if UL formulation is used

more convenient, see Reference [38]. Equation (52) may be rearranged as

$$
{ }_{t}^{t+\Delta t} \mathbf{R}^{w} \mathbf{E}_{*}^{\mathrm{e} t+\Delta t} \mathbf{R}_{t}^{w \mathrm{~T}} \simeq{ }_{0}^{t+\Delta t} \mathbf{E}^{\mathrm{e}}+\Delta t{ }^{t+\Delta t} \overline{\mathbf{D}}^{\mathrm{p}}
$$

and using ${ }^{t+\Delta t} \mathbf{R}^{w} \simeq \mathbf{I}+\Delta t{ }^{t+\Delta t} \overline{\mathbf{W}}^{\mathrm{p}}$ we obtain

$$
{ }^{t+\Delta t} \mathbf{E}^{\mathrm{e}} \simeq \mathbf{E}_{*}^{\mathrm{e}}-\Delta t{ }^{t+\Delta t} \overline{\mathbf{D}}^{\mathrm{p}}-\Delta t{ }^{t+\Delta t} \boldsymbol{\Theta}
$$

where ${ }^{t+\Delta t} \boldsymbol{\Theta}$ is the commutation tensor between $\mathbf{E}_{*}^{\mathrm{e}}$ and ${ }^{t+\Delta t} \overline{\mathbf{W}}^{\mathrm{p}}$ defined as

$$
{ }^{t+\Delta t} \boldsymbol{\Theta}:=\mathbf{E}_{*}^{\mathrm{e} t+\Delta t} \overline{\mathbf{W}}^{\mathrm{p}}-{ }^{t+\Delta t} \overline{\mathbf{W}}^{\mathrm{p}} \mathbf{E}_{*}^{\mathrm{e}}
$$

Equation (62) also preserves the convenient additive incremental decomposition of (logarithmic) strains.

\subsection{Isotropic hardening and mixed hardening without and with plastic spin}

Some cases are of special interest and have been studied extensively in the literature. The first case is isotropic hardening without plastic spin. In this simplest case which was proposed by Weber and Anand [20] the principal directions of the trial strain (stress) tensor and the final strain tensor are coincident. Thus ${ }_{0}^{t+\Delta t} \tilde{\mathbf{E}}^{\mathrm{e}}={ }^{t+\Delta t} \mathbf{E}^{\mathrm{e}}$ and ${ }^{t+\Delta t} \mathbf{R}^{\mathrm{e}} \simeq \mathbf{R}_{*}^{\mathrm{e}}$. Furthermore, since the principal directions are fixed during the plastic flow, the return may be performed in the principal directions space, i.e. reducing the dimension of the problem to three. In this setting we define $\hat{\tau}^{\text {princ }}:=\left[\tau_{1}, \tau_{2}, \tau_{3}\right]^{\mathrm{T}}$ as the vector of principal stress values and perform the integration using this vector, instead of using the full stress vector $\hat{\tau}:=\left[\tau_{11}, \tau_{22}, \tau_{33}, \tau_{12}, \tau_{23}, \tau_{13}\right]^{\mathrm{T}}$. Also, since $\mathbf{R}^{\mathrm{e}}$ plays no role in the formulation, it is sufficient to store only the elastic Finger tensor instead of the plastic or elastic deformation gradient as shown by Simó [22]. 
The second case is kinematic hardening when the plastic spin is zero. This formulation was presented by Eterović and Bathe [21]. In such cases the formulation simplifies considerably during the stress-integration phase since ${ }_{0}^{t+\Delta t} \tilde{\mathbf{E}}^{\mathrm{e}}={ }_{0}^{t+\Delta t} \mathbf{E}^{\mathrm{e}}$ and ${ }^{t+\Delta t}{ }_{0} \mathbf{R}^{\mathrm{e}} \simeq \mathbf{R}_{*}^{\mathrm{e}}$. However, it is necessary to note that in general anisotropic kinematic (or combined) hardening the principal directions do not remain constant during the plastic flow, as a simple inspection of the update formula (52) shows, since, as shown below, the principal directions of the backstress and the rotated stress tensors are not, in general, coincident.

Given that for the isotropic hardening case the principal directions of the stress tensor remain constant in an incremental step during the plastic flow, one may be tempted to extend this setting to the kinematic/mixed hardening formulation in order to simplify the numerical algorithm using the following assumptions:

\section{- Assumption 1}

The final stresses have the same principal directions as the trial stresses even for the kinematic hardening case.

- Assumption 2

In addition to using Assumption 1, we simplify the model further by performing the integration in a space of reduced dimension, using only the principal stress values, i.e. the vectors $\hat{\tau}^{\text {princ }}=\left[\tau_{1}, \tau_{2}, \tau_{3}\right]^{\mathrm{T}}$ for the actual stresses and $\hat{\boldsymbol{\beta}}^{\text {princ }}=\left[\beta_{1}, \beta_{2}, \beta_{3}\right]^{\mathrm{T}}$ for the backstresses. This implies that the principal directions of the trial backstress tensor are also the same as those of the trial stress tensor.

This simplified setting, with both assumptions, was considered in Reference [22], whereas in Reference [24] the authors used Assumption 1 but using the full stress space for the plastic return. However, these assumptions have some consequences in the constitutive model and in the predictions obtained.

3.2.1. Consequences of Assumption 1. The first assumption includes inadvertently a plastic spin for the general kinematic/mixed hardening cases. This plastic spin is given by the requirement that the eigenvectors of ${ }_{0}^{t+\Delta t} \mathbf{E}^{\mathrm{e}}$ (or ${ }^{t+\Delta t} \bar{\tau}$ ) are the same as those of $\mathbf{E}_{*}^{\mathrm{e}}$. Thus, denoting by $\mathbf{N}_{i}^{*}$, ${ }^{t+\Delta t} \tilde{\mathbf{N}}_{i}$ and ${ }^{t+\Delta t} \mathbf{N}_{i}$ the eigenvectors of $\mathbf{E}_{*}^{\mathrm{e}},{ }^{t+\Delta t} \tilde{\mathbf{E}}^{\mathrm{e}}$ and ${ }_{0}^{t+\Delta t} \mathbf{E}^{\mathrm{e}}$, respectively, the requirement is

$$
{ }^{t+\Delta t} \mathbf{N}_{i} \equiv{ }^{t+\Delta t}{ }_{t} \mathbf{R}^{w+\Delta t} \tilde{\mathbf{N}}_{i}=\mathbf{N}_{i}^{*}
$$

so the incremental plastic rotation is obtained by the explicit expression

$$
{ }^{t+\Delta t}{ }_{t} \mathbf{R}^{w}=\sum_{i=1}^{3} \mathbf{N}_{i}^{*} \otimes{ }^{t+\Delta t} \tilde{\mathbf{N}}_{i}
$$

and the plastic spin is inherently given by

$$
{ }^{t+\Delta t} \overline{\mathbf{W}}^{\mathrm{p}}=\frac{1}{\Delta t} \ln \left({ }^{t+\Delta t} \mathbf{R}^{w}\right)
$$

Let us denote by $\boldsymbol{\Omega}^{N *}$ the spin of the trial basis (i.e. 'freezing' the plastic flow) such that $\dot{\mathbf{N}}_{i}^{*}=\mathbf{\Omega}^{N *} \mathbf{N}_{i}^{*}$ and by ${ }^{t+\Delta t} \mathbf{\Omega}^{\tilde{N}}$ the spin of the eigenbasis of ${ }^{t+\Delta t} \overline{\mathbf{T}}$ at $t+\Delta t$ such 
that ${ }^{t+\Delta t} \dot{\tilde{\mathbf{N}}}_{i}={ }^{t+\Delta t} \mathbf{\Omega}^{\tilde{N}}{ }^{t+\Delta t} \tilde{\mathbf{N}}_{i}$. Then

$$
{ }^{t+\Delta t} \boldsymbol{\Omega}^{\tilde{N}}:=\sum_{i=1}^{3}{ }^{t+\Delta t} \dot{\tilde{\mathbf{N}}}_{i} \otimes{ }^{t+\Delta t} \tilde{\mathbf{N}}_{i} \quad \text { and } \quad \mathbf{\Omega}^{N *}:=\sum_{i=1}^{3} \dot{\mathbf{N}}_{i}^{*} \otimes \mathbf{N}_{i}^{*}
$$

and the plastic spin may be obtained from (65) and a simple inspection of Equations (39) and (40) also as

$$
{ }^{t+\Delta t} \overline{\mathbf{W}}^{\mathrm{p}}={ }^{t+\Delta t}{ }_{t} \dot{\mathbf{R}}^{w}{ }_{t}^{t+\Delta t} \mathbf{R}^{w \mathrm{~T}}=\mathbf{\Omega}^{N *}+{ }^{t+\Delta t}{ }_{t} \mathbf{R}^{w}\left({ }^{t+\Delta t} \mathbf{\Omega}^{\tilde{N}}\right){ }^{\mathrm{T}}{ }_{t+\Delta t} \mathbf{R}^{w \mathrm{~T}}
$$

In the limit case when $\Delta t \rightarrow 0$ we have ${ }^{t+\Delta t}{ }_{t} \mathbf{R}^{w}=\mathbf{I}+\Delta t{ }^{t+\Delta t} \overline{\mathbf{W}}^{\mathrm{p}} \rightarrow \mathbf{I}$ and ${ }^{t} \overline{\mathbf{W}}^{\mathrm{p}}=\mathbf{\Omega}^{N *}-{ }^{t} \mathbf{\Omega}^{\tilde{N}}$, which is the continuum value for this particular formulation. Therefore the spin tensor for the vectors ${ }^{t} \mathbf{N}_{i}$ is ${ }^{t} \boldsymbol{\Omega}^{N} \equiv{ }^{t} \boldsymbol{\Omega}^{\tilde{N}}+{ }^{t} \overline{\mathbf{W}}^{\mathrm{p}}=\boldsymbol{\Omega}^{N *}$ and, as expected, we have that the stress tensor rotates in the same way as the trial stress tensor. This spin produces a special kind of anisotropic kinematic hardening, different from the usual one as already mentioned in Reference [24]. As shown in the examples below, the predicted behaviour under non-proportional loading is different from that obtained with the usual kinematic hardening model.

3.2.2. Consequences of Assumption 2. The use of Assumption 2 also introduces inadvertently a rotation to the backstress. This hidden rotation takes place even during purely elastic deformations, but affects the stresses only during plastic flow (and therefore it can be viewed as some sort of plastic spin). To understand the reason of this additional rotation it is sufficient to consider two consecutive solution steps.

Let ${ }^{t} \mathbf{X}^{\mathrm{e}}$ be the final, converged, elastic deformation gradient at the end of step $t$ consisting of a pure stretch tensor ${ }^{t} \mathbf{U}^{\mathrm{e}}$. For simplicity we consider a $2 \mathrm{D}$ case

$$
{ }^{t} \mathbf{X}^{\mathrm{e}}={ }^{t} \mathbf{U}^{\mathrm{e}}=\left[\begin{array}{ll}
\lambda & \\
& 1 / \lambda
\end{array}\right]
$$

The eigenvectors of this stretch tensor are

$$
{ }^{t} \mathbf{N}_{1}=[1,0]^{\mathrm{T}}, \quad{ }^{t} \mathbf{N}_{2}=[0,1]^{\mathrm{T}}
$$

Consider now that the trial deformation gradient $\mathbf{X}_{*}^{\mathrm{e}}:={ }^{t+\Delta t}{ }_{t}^{t} \mathbf{X}^{t} \mathbf{X}^{\mathrm{e}}$ for the step $t+\Delta t$ is also a pure stretch tensor $\mathbf{U}_{*}^{\mathrm{e}}$, with a different eigenbasis

$$
\mathbf{X}_{*}^{\mathrm{e}}=\mathbf{U}_{*}^{\mathrm{e}}=\left[\begin{array}{ll}
a & b \\
b & a
\end{array}\right]
$$

If $b= \pm \sqrt{a^{2}-1}$ the tensor is isochoric. Of course depending on the values of $a, b$ the incremental response may still be elastic. For instance, the values $a=\frac{1}{2}(\lambda+1 / \lambda)$ and $b=$ $\frac{1}{2}(\lambda-1 / \lambda)$ give for (71) the same eigenvalues as those of (69). The incremental deformation gradient is ${ }^{t+\Delta t} \mathbf{X}=\mathbf{U}_{*}^{\mathrm{e} t} \mathbf{U}^{\mathrm{e}-1}$ and the trial eigenvectors $\mathbf{N}_{i}^{*}$ differ from ${ }^{t} \mathbf{N}_{i}$, i.e. for (71) we have

$$
\mathbf{N}_{1}^{*}=\frac{1}{\sqrt{2}}[1,1]^{\mathrm{T}}, \quad \mathbf{N}_{2}^{*}=\frac{1}{\sqrt{2}}[-1,1]^{\mathrm{T}}
$$

The (elastic) rotation tensors of the solid at both time steps are ${ }^{t} \mathbf{R}^{\mathrm{e}}=\mathbf{R}_{*}^{\mathrm{e}}=\mathbf{I}$. If the solution is performed using the principal trial stress directions for the stress and the backstresses, the 
trial backstress eigenvectors at $t+\Delta t$ are given by $\mathbf{N}_{i}^{*}$ instead of the correct ${ }^{t} \mathbf{N}_{i}$. Namely, the backstress at the end of step $t$ was

$$
{ }^{t} \overline{\boldsymbol{\beta}}=\sum_{i=1}^{2}{ }^{t} \beta_{i}{ }^{t} \mathbf{N}_{i} \otimes{ }^{t} \mathbf{N}_{i}=\left[\begin{array}{ll}
{ }^{t} \beta_{1} & \\
& { }^{t} \beta_{2}
\end{array}\right]
$$

Of course since ${ }^{t} \overline{\boldsymbol{\beta}}$ is deviatoric in nature, ${ }^{t} \beta_{2}=-{ }^{t} \beta_{1}$ for the $2 \mathrm{D}$ case. The trial backstress for step $t+\Delta t$, which by definition should be $\overline{\boldsymbol{\beta}}_{*}={ }^{t} \overrightarrow{\boldsymbol{\beta}}$, takes instead the value

$$
\overline{\boldsymbol{\beta}}_{*}=\sum_{i=1}^{2}{ }^{t} \beta_{i} \mathbf{N}_{i}^{*} \otimes \mathbf{N}_{i}^{*}=\frac{1}{2}\left[\begin{array}{cc}
{ }^{t} \beta_{1}+{ }^{t} \beta_{2} & { }^{t} \beta_{1}-{ }^{t} \beta_{2} \\
{ }^{t} \beta_{1}-{ }^{t} \beta_{2} & { }^{t} \beta_{1}+{ }^{t} \beta_{2}
\end{array}\right]=\left[\begin{array}{cc}
0 & { }^{t} \beta_{1} \\
{ }^{t} \beta_{1} & 0
\end{array}\right]
$$

That is to say, although the eigenvalues are the same, the backstress has rotated the amount

$$
{ }_{t}^{t+\Delta t} \mathbf{R}^{\boldsymbol{\beta}}=\sum_{i=1}^{2} \mathbf{N}_{i}^{*} \otimes{ }^{t} \mathbf{N}_{i}=\frac{1}{\sqrt{2}}\left[\begin{array}{cc}
1 & -1 \\
1 & 1
\end{array}\right]
$$

so that $\overline{\boldsymbol{\beta}}_{*}={ }^{t+\Delta t}{ }_{t} \mathbf{R}^{\boldsymbol{\beta}} \overline{\boldsymbol{\beta}}\left({ }^{t+\Delta t} \mathbf{R}^{\boldsymbol{\beta}}\right)^{\mathrm{T}}$. This effect was already noted in Reference [39], where also more results are reported using a spatial algorithm and a Finger tensor based algorithm. We note that this backstress rotation affects both the backstress and stress evolution during the plastic return, since the backstress rotation takes place over the trial value. However, it is only due to the trial stress rotation. Thus, it seems difficult to formulate a physical characterization of this spin and a constitutive model that explicitly accounts for this spin.

3.2.3. Example. In order to exemplify the concepts discussed above, consider a $2 \mathrm{D}$ example with two consecutive load steps. The (isochoric) deformation gradient for the first step consists of the pure stretch tensor:

$$
{ }^{1} \mathbf{X}={ }^{1} \mathbf{X}_{*}^{\mathrm{e}}={ }^{1} \mathbf{U}_{*}^{\mathrm{e}}=\left[\begin{array}{cc}
2 & \\
& \\
& \frac{1}{2}
\end{array}\right]
$$

Consider as material constants $\mu=5, H^{\prime}=15$ (a linear kinematic) and $\sigma_{y}=4$ as yield stress. The trial stresses and backstresses are

$$
{ }^{1} \overline{\mathbf{T}}_{*} \equiv \bar{\tau}_{*}=2 \mu \ln { }^{1} \mathbf{U}_{*}^{\mathrm{e}}=\left[\begin{array}{cc}
6.932 & 0 \\
0 & -6.932
\end{array}\right], \quad{ }^{1} \overline{\mathbf{B}}_{*}=\mathbf{0}
$$

For the Mises yield function $f:=\|\overline{\mathbf{T}}-\overline{\mathbf{B}}\|-\sqrt{\frac{3}{2}} \sigma_{y}$, the flow direction and consistency parameter increment are

$$
{ }^{1} \overline{\mathbf{D}}^{\mathrm{p}}=\frac{\partial f}{\partial \overline{\mathbf{T}}}=\frac{{ }^{1} \overline{\mathbf{T}}_{*}-{ }^{1} \overline{\mathbf{B}}_{*}}{\left\|{ }^{1} \overline{\mathbf{T}}_{*}-{ }^{1} \overline{\mathbf{B}}_{*}\right\|}=\frac{1}{\sqrt{2}}\left[\begin{array}{cc}
1 & 0 \\
0 & -1
\end{array}\right], \quad{ }^{1} \Delta t=\frac{f\left({ }^{1} \overline{\mathbf{T}}_{*}-{ }^{1} \overline{\mathbf{B}}_{*}\right)}{2 \mu+\frac{2}{3} H^{\prime}}=0.2452
$$


Therefore, the final solution for the step is

$$
\begin{gathered}
{ }^{1} \overline{\mathbf{T}} \equiv{ }^{1} \bar{\tau}={ }^{1} \overline{\mathbf{T}}_{*}-2 \mu^{1} \Delta t{ }^{1} \overline{\mathbf{D}}^{\mathrm{p}}=\left[\begin{array}{cc}
5.198 & 0 \\
0 & -5.198
\end{array}\right] \\
{ }^{1} \overline{\mathbf{B}} \equiv{ }^{1} \overline{\boldsymbol{\beta}}={ }^{1} \mathbf{B}_{*}+{ }^{2}{ }_{3} H^{\prime}{ }^{1} \Delta t{ }^{1} \overline{\mathbf{D}}^{\mathrm{p}}=\left[\begin{array}{cc}
1.734 & 0 \\
0 & -1.734
\end{array}\right]
\end{gathered}
$$

We consider now that the trial (isochoric) elastic deformation gradient for the second step is

$$
{ }^{2} \mathbf{X}_{*}^{\mathrm{e}}={ }^{2} \mathbf{U}_{*}^{\mathrm{e}}=\left[\begin{array}{ll}
1.6667 & 1.3333 \\
1.3333 & 1.6667
\end{array}\right]
$$

The trial stresses and backstresses are

$$
{ }^{2} \overline{\mathbf{T}}_{*}=2 \mu \ln { }^{2} \mathbf{U}_{*}^{\mathrm{e}}=\left[\begin{array}{cc}
0 & 10.986 \\
10.986 & 0
\end{array}\right], \quad{ }^{2} \overline{\mathbf{B}}_{*}={ }^{1} \overline{\boldsymbol{\beta}}=\left[\begin{array}{cc}
1.734 & 0 \\
0 & -1.734
\end{array}\right]
$$

The stresses ${ }^{2} \overline{\mathbf{T}}_{*}$ have the eigenvectors

$$
{ }^{2} \mathbf{N}_{1}^{*}=\frac{1}{\sqrt{2}}\left[\begin{array}{ll}
1 & 1
\end{array}\right]^{\mathrm{T}} \text { and }{ }^{2} \mathbf{N}_{2}^{*}=\frac{1}{\sqrt{2}}\left[\begin{array}{ll}
1 & -1
\end{array}\right]^{\mathrm{T}}
$$

The solution, following the same procedure as for step 1 , is

$$
\begin{aligned}
{ }^{2} \tilde{\mathbf{D}}^{\mathrm{p}}=\left[\begin{array}{cc}
-0.110 & 0.698 \\
0.698 & 0.110
\end{array}\right], \quad{ }^{2} \Delta t=0.541 \\
{ }^{2} \overline{\mathbf{T}}=\left[\begin{array}{cc}
0.597 & 7.204 \\
7.204 & -0.597
\end{array}\right], \quad{ }^{2} \overline{\mathbf{B}}=\left[\begin{array}{cc}
1.137 & 3.782 \\
3.782 & -1.137
\end{array}\right]
\end{aligned}
$$

If vanishing plastic spin is assumed, the solution is ${ }^{2} \bar{\tau}={ }^{2} \overline{\mathbf{T}}$ and ${ }^{2} \overline{\boldsymbol{\beta}}={ }^{2} \overline{\mathbf{B}}$, as performed in Reference [21].

If Assumption 1 is enforced, then we proceed to check the eigenvectors of ${ }^{2} \overline{\mathbf{T}}$ and see that they differ from those of ${ }^{2} \overline{\mathbf{T}}_{*}$ :

$$
{ }^{2} \tilde{\mathbf{N}}_{1}=\left[\begin{array}{ll}
0.736 & 0.677
\end{array}\right]^{\mathrm{T}} \text { and }{ }^{2} \tilde{\mathbf{N}}_{2}=\left[\begin{array}{ll}
0.677 & -0.736
\end{array}\right]^{\mathrm{T}}
$$

Hence, we apply the incremental plastic rotation

$$
{ }_{1}^{2} \mathbf{R}^{w}=\sum_{i=1}^{3}{ }^{2} \mathbf{N}_{i}^{*} \otimes{ }^{2} \tilde{\mathbf{N}}_{i}=\left[\begin{array}{cc}
0.99915 & -0.04133 \\
0.04133 & 0.99915
\end{array}\right]
$$

to obtain

$$
{ }^{2} \overline{\boldsymbol{\tau}}={ }_{1}^{2} \mathbf{R}^{w}{ }^{2} \overline{\mathbf{T}}_{1}^{2} \mathbf{R}^{w \mathrm{~T}}=\left[\begin{array}{cc}
0 & 7.228 \\
7.228 & 0
\end{array}\right], \quad{ }^{2} \overline{\boldsymbol{\beta}}={ }_{1}^{2} \mathbf{R}^{w 2} \overline{\mathbf{B}}_{1}^{2} \mathbf{R}^{w \mathrm{~T}}=\left[\begin{array}{cc}
0.821 & 3.863 \\
3.863 & -0.821
\end{array}\right]
$$


We note that in this case the eigenvectors of ${ }^{2} \bar{\tau}$ are ${ }^{2} \mathbf{N}_{i}^{*}$ (we have enforced it) but those of ${ }^{2} \overline{\boldsymbol{\beta}}$ take on, in general, different values:

$$
{ }^{2} \mathbf{N}_{1}^{\beta}=\left[\begin{array}{ll}
0.777 & 0.630
\end{array}\right]^{\mathrm{T}} \text { and }{ }^{2} \mathbf{N}_{2}^{\beta}=\left[\begin{array}{lll}
0.630 & -0.777
\end{array}\right]^{\mathrm{T}}
$$

If Assumption 2 is enforced, the trial backstress in Equation (82) rotates in order to have the same eigenvectors as those of ${ }^{2} \overline{\mathbf{T}}_{*}$. The value of these backstresses are

$$
{ }^{2} \overline{\boldsymbol{\beta}}_{*}=\sum_{i=1}^{2}{ }^{1} \beta_{i}{ }^{2} \mathbf{N}_{i}^{*} \otimes{ }^{2} \mathbf{N}_{i}^{*}=\left[\begin{array}{cc}
0 & 1.734 \\
1.734 & 0
\end{array}\right]
$$

Hence, the results from Equations (82) to (88) are different (note that the stresses have the same direction as with Assumption 1, but differ in magnitude)

$$
{ }^{2} \overline{\mathbf{D}}^{\mathrm{p}}=\frac{1}{\sqrt{2}}\left[\begin{array}{ll}
0 & 1 \\
1 & 0
\end{array}\right], \quad{ }^{2} \Delta t=0.409, \quad{ }^{2} \bar{\tau}=\left[\begin{array}{cc}
0 & 8.091 \\
8.091 & 0
\end{array}\right], \quad{ }^{2} \overline{\boldsymbol{\beta}}=\left[\begin{array}{cc}
0 & 4.628 \\
4.628 & 0
\end{array}\right]
$$

Of course in this case the trial stress, trial backstress, final stress and final backstress tensors have all the same eigenvectors. In fact the solution could have been performed using only the eigenvalues.

In Figure 2 the integration process for the algorithms considered here is illustrated. In this figure we (heuristically) represent tensors by 2D vectors with one 'principal direction' given by the direction of the vector and one 'principal value' given by its modulus. It is seen that all algorithms would give different results. It is remarkable that the backstress rotation, that takes place in the integration algorithm performed in the reduced space, results in an effective yield surface (the one detected using the test on the trial yield function ${ }^{2} f_{*}=0$ for all the possible trial states) similar to that obtained using isotropic hardening, as shown in Figure 2(e). In the six-dimensional space a similar effect is observed, although, since in this case negative eigenvalues are possible, a stress reversal would result in plastic flow. The practical consequence of these observations is illustrated in Section 5, especially in the numerical examples under cyclic shearing.

We finally note that both special types of kinematic/mixed hardening could also be performed in infinitesimal strain analysis, but such approaches are not a common choice. Hence, the selection of this model in finite deformation analysis seems to be based only on numerical simplicity. The more general formulation presented in this paper also allows for the use of anisotropic yield functions. Note that no assumption has been made so far on the shape of the yield function and that, frequently, the elastic properties are considered isotropic, even in the case of anisotropic plastic properties developed in cold-worked metals, see for example References [40,41] and for an experimental confirmation [33,42]. If elastic anisotropic properties are to be modelled, some modifications to the algorithm are necessary, but the kinematics of the formulation remains essentially the same, see Reference [38]. Also, the more 

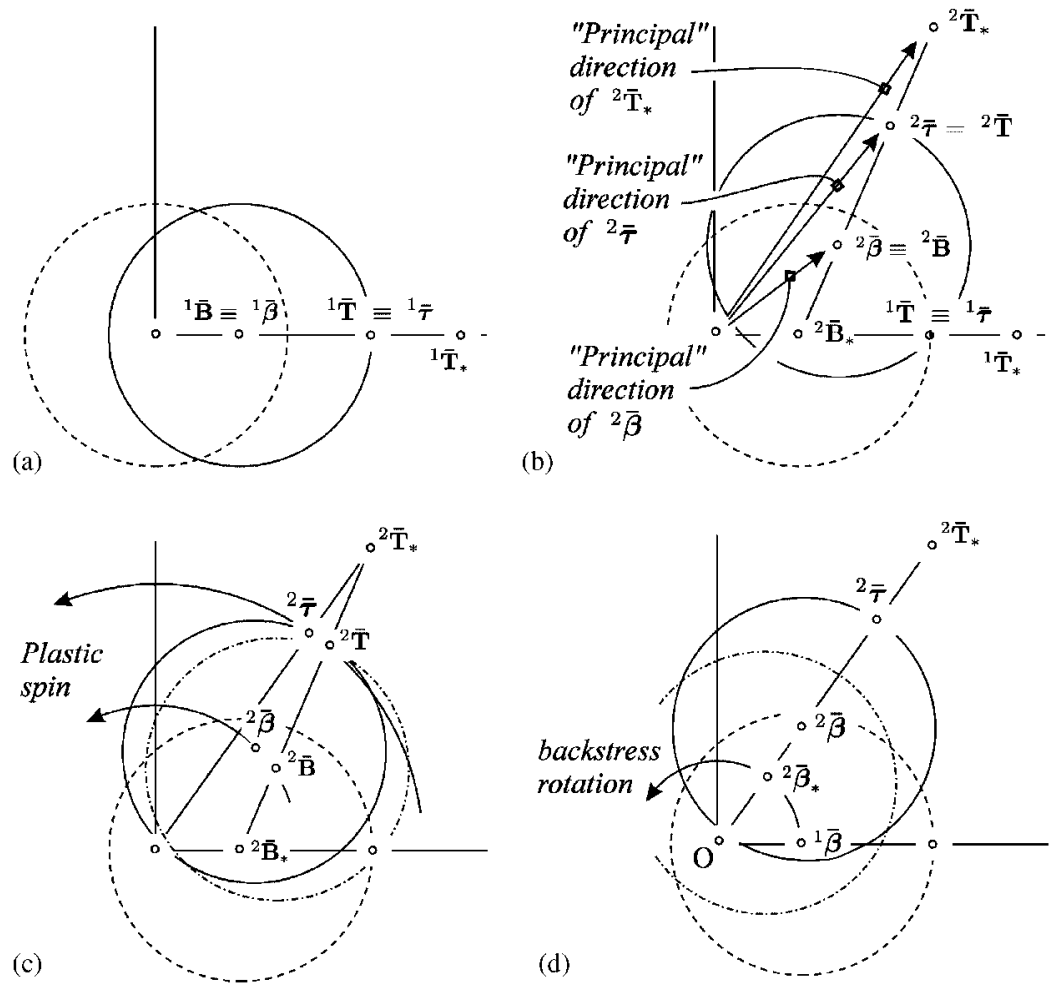

(d)

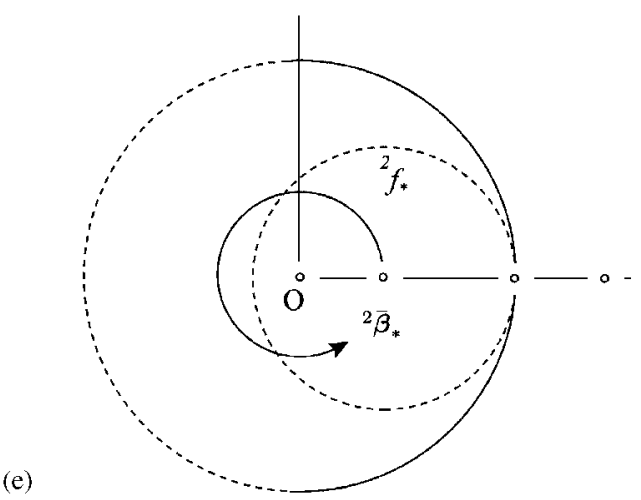

Figure 2. Evolution of the stresses and backstresses for the 'models' without and with Assumptions 1 and 2: (a) Step 1-all models; (b) step 2-usual kinematic hardening without plastic spin; (c) step 2 -kinematic hardening with plastic spin; (d) step 2-Integration in the reduced space given by the line $\mathrm{O}-{ }^{2} \overline{\mathrm{T}}_{*}$; and (e) step 2-'effective' yield surface for integration in reduced space; obtained by rotating the trial yield surface ${ }^{2} f_{*}$ about the origin $\mathrm{O}$.

sophisticated implicit multilayer kinematic hardening models [43,44] or bounding surface models [45] for a better description of cyclic plastic behaviour can be inserted in the formulation with ease. 


\section{ALGORITHMIC CONSISTENT TANGENT MODULI}

Although a numerically evaluated algorithmic constitutive tangent is possible by means of a perturbation process of the stress-integration algorithm as shown in References [46,47], the development of the (analytical) consistent tangent moduli should not be regarded simply as an important computational convenience to (possibly) reduce the computational time. The derivation also allows a more detailed analysis of the algorithm itself, which frequently gives further insight into the formulation.

\subsection{General case}

During the iterative process, the trial elastic state changes as shown in Figure 3. The updated co-ordinates are

$$
{ }^{t+\Delta t} \mathbf{x}^{(i+1)}={ }^{t+\Delta t} \mathbf{x}^{(i)}+\zeta \mathbf{u}_{(i)}^{(i+1)}
$$

where $\zeta$ is a parameter which has the value $\zeta=1$ in Equation (91) and $\mathbf{u}_{(i)}^{(i+1)}$ is the increment between iterations. The total and trial deformation gradient tensors may be expressed as (just following the same steps to obtain Equation (5))

$$
\begin{aligned}
{ }_{0}^{t+\Delta t} \mathbf{X}^{(i+1)} & =\mathbf{X}_{(i)}^{(i+1) t+\Delta t}{ }_{0}^{(i)} \\
\mathbf{X}_{*}^{\mathrm{e}(i+1)} & =\mathbf{X}_{(i)}^{(i+1)} \mathbf{X}_{*}^{\mathrm{e}(i)}
\end{aligned}
$$

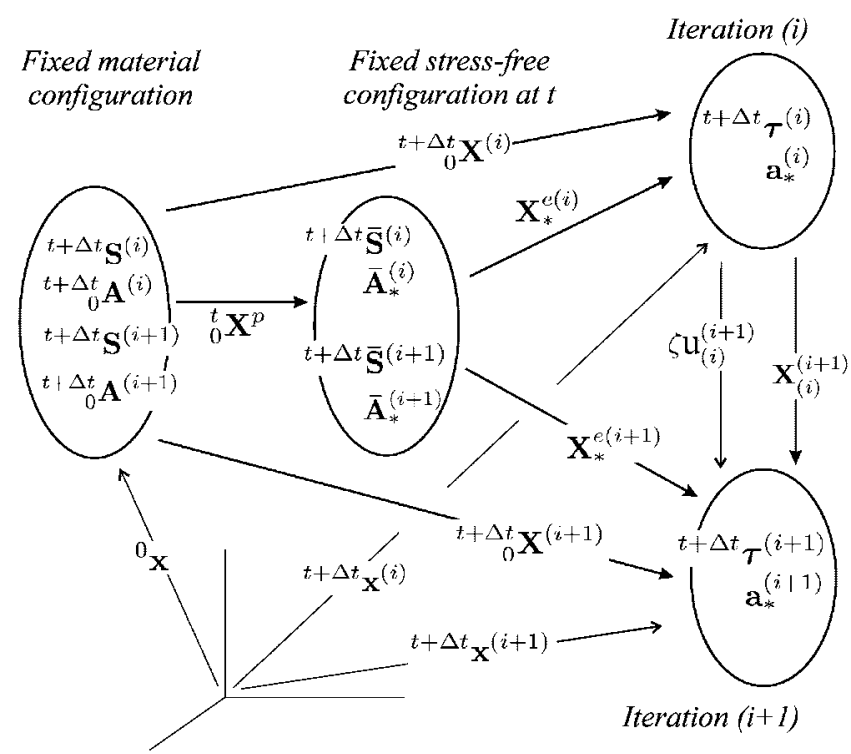

Figure 3. Main configurations involved in the linearization of the algorithm around iteration $(i)$. 
where $\mathbf{X}_{(i)}^{(i+1)}=\mathbf{I}+\zeta \nabla_{(i)} \mathbf{u}_{(i)}^{(i+1)}$ and $\nabla_{(i)}=\partial / \partial^{t+\Delta t} \mathbf{x}^{(i)}$ denotes the gradient with respect to the configuration for iteration $(i)$. In the finite element formulation we wish to obtain the tensor ${ }^{t+\Delta t} \mathbb{C}$ such that (see Reference [32])

$$
J^{-1} \mathscr{L}_{\Delta}^{(i)}{ }^{t+\Delta t} \tau^{(i)}=J^{-1 t+\Delta t} \mathbb{C}^{(i)}: \nabla_{(i)}^{s}{ }^{t+\Delta t} \mathbf{u}^{(i+1)}
$$

where $\nabla_{(i)}^{s}$ is the symmetric gradient and $\mathscr{L}_{\Delta}^{(i)}(\cdot)$ is the incremental Lie derivative at iteration (i) defined for contravariant tensors as

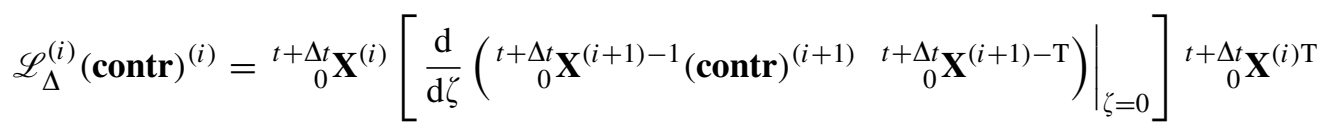

and for covariant tensors as

$$
\mathscr{L}_{\Delta}^{(i)}(\mathbf{c o v})^{(i)}={ }_{0}^{t+\Delta t} \mathbf{X}^{(i)-\mathrm{T}}\left[\left.\frac{\mathrm{d}}{\mathrm{d} \zeta}\left({ }_{0}^{t+\Delta t} \mathbf{X}^{(i+1) \mathrm{T}}(\mathbf{c o v})^{(i+1)}{ }_{0}^{t+\Delta t} \mathbf{X}^{(i+1)}\right)\right|_{\zeta=0}\right]{ }_{0}^{t+\Delta t} \mathbf{X}^{(i)-1}
$$

Given a preimposed fixed (during the iteration process) plastic deformation tensor ${ }_{0}^{t} \mathbf{X}^{\mathrm{p}}$, it is easily checked that since ${ }_{0}^{t+\Delta t} \mathbf{X}^{(i+1)}=\mathbf{X}_{*}^{\mathrm{e}(i+1)}{ }_{0}^{t} \mathbf{X}^{\mathrm{p}}$, expressions (95) and (96) may be used employing either ${ }_{0}^{t+\Delta t} \mathbf{X}$ or $\mathbf{X}_{*}^{\mathrm{e}}$; this fact follows also from a comparison of Equations (92) and (93).

Defining the trial elastic Almansi strain tensor as

$$
\mathbf{a}_{*}=\frac{1}{2}\left(\mathbf{I}-\mathbf{X}_{*}^{\mathrm{e}-\mathrm{T}} \mathbf{X}_{*}^{\mathrm{e}-1}\right)
$$

and the pull-back to the stress free configuration as $\overline{\mathbf{A}}_{*}^{(i+1)}=\frac{1}{2}\left(\mathbf{X}_{*}^{\mathrm{e}(i) \mathrm{T}} \mathbf{X}_{(i)}^{(i+1) \mathrm{T}} \mathbf{X}_{(i)}^{(i+1)} \mathbf{X}_{*}^{\mathrm{e}(i)}-\mathbf{I}\right)$, the Lie derivative for the current one is (see Reference [32] for a different but equivalent approach)

$$
\mathscr{L}_{\Delta}^{(i)} \mathbf{a}_{*}^{(i)}=\mathbf{X}_{*}^{\mathrm{e}(i)-\mathrm{T}}\left[\left.\frac{\mathrm{d}}{\mathrm{d} \zeta} \overline{\mathbf{A}}_{*}^{(i+1)}\right|_{\zeta=0}\right] \mathbf{X}_{*}^{\mathrm{e}(i)-1}=\nabla_{(i)}^{s}{ }^{t+\Delta t} \mathbf{u}^{(i+1)}
$$

i.e. $\mathbf{d}^{(i)}:=\mathscr{L}_{\Delta}^{(i)} \mathbf{a}^{(i)}=\mathscr{L}_{\Delta}^{(i)} \mathbf{a}_{*}^{(i)}$.

Furthermore, given a superimposed arbitrary rotation $\mathbf{Q}$, it is easily seen that,

$$
\mathscr{L}_{\Delta}^{(i)}(\cdot)^{(i)}=\mathbf{Q}^{\mathrm{T}} \mathscr{L}_{Q}^{(i)}\left(\mathbf{Q}(\cdot) \mathbf{Q}^{\mathrm{T}}\right)^{(i)} \mathbf{Q}
$$

where $\mathscr{L}_{Q}^{(i)}(\cdot)^{(i)}$ coincides with (95) or (96) upon substitution of ${ }_{0}^{t+\Delta t} \mathbf{X}$ by ${ }_{0}^{t+\Delta t} \tilde{\mathbf{X}}=\mathbf{Q}_{0}^{t+\Delta t} \mathbf{X}$. Common meaningful choices are, as already mentioned, $\mathbf{Q}=\mathbf{R}_{*}^{\mathrm{eT}}, \mathbf{Q}=\mathbf{I}$ and $\mathbf{Q}=\mathbf{R}_{*}^{\text {princT }}$. Hence, it is simply necessary to obtain the rotated tensor ${ }^{t+\Delta t} C_{Q}^{(i) i j k l}=Q_{a .}^{i} Q_{b .}^{\cdot j} Q_{c .}^{\cdot k} Q_{d}^{. l}$ ${ }^{t+\Delta t} C^{(i) a b c d}$ such that

$$
\mathscr{L}_{Q}^{(i)}\left({ }^{t+\Delta t} \bar{\tau}^{(i)}\right)={ }^{t+\Delta t} \mathbb{C}_{Q}^{(i)}: \mathscr{L}_{Q}^{(i)}\left({ }^{t+\Delta t} \overline{\mathbf{a}}_{*}^{(i)}\right)
$$

where ${ }^{t+\Delta t} \bar{\tau}^{(i)}=\mathbf{Q}^{t+\Delta t} \tau^{(i)} \mathbf{Q}^{\mathrm{T}}$ and ${ }^{t+\Delta t} \overline{\mathbf{a}}_{*}^{(i)}=\mathbf{Q}^{t+\Delta t} \mathbf{a}_{*}^{(i)} \mathbf{Q}^{\mathrm{T}}$ are the rotated quantities. In order to simplify the notation we will denote derivatives with respect to the parameter $\zeta$ as time 
derivatives and the iteration index $(i)$ will be omitted. Also, to keep the formulation simple and concrete, we consider the case $\mathbf{Q}=\mathbf{R}_{*}^{\mathrm{eT}}$, but the same procedure applies to any other case.

The Lie derivative of the rotated stress tensor and the rotated Almansi strains may be written as

$$
\mathscr{L}_{Q}^{(i)}\left({ }^{t+\Delta t} \bar{\tau}\right)=\mathbf{U}_{*}^{\mathrm{e} t+\Delta t} \dot{\overline{\mathbf{S}}} \mathbf{U}_{*}^{\mathrm{e}}, \quad \mathscr{L}_{Q}^{(i)}\left({ }^{t+\Delta t} \overline{\mathbf{a}}_{*}\right)=\mathbf{U}_{*}^{\mathrm{e}-1} \dot{\overline{\mathbf{A}}}_{*} \mathbf{U}_{*}^{\mathrm{e}-1}
$$

where ${ }^{t+\Delta t} \overline{\mathbf{S}}$ is the pull-back of the spatial Kirchhoff stress tensor (and of the rotated one) to the intermediate stress-free configuration. It is noted that the pull-back by $\mathbf{X}_{*}^{\mathrm{e}}$ of the eigenvectors of ${ }^{t+\Delta t} \tau$ do not necessarily form an orthogonal basis in the Euclidean metric. In view of (101), the tensor ${ }^{t+\Delta t} C_{Q}^{i j k l}$ may be obtained as the push forward by $\mathbf{U}_{*}^{\mathrm{e}}$ of the tensor ${ }^{t+\Delta t} \overline{\mathbb{C}}$ such that

$$
{ }^{t+\Delta t} \dot{\overline{\mathbf{S}}}={ }^{t+\Delta t} \overline{\mathbb{C}}: \dot{\overline{\mathbf{A}}}_{*}
$$

Note that if $\mathbf{Q}=\mathbf{R}_{*}^{\mathrm{eT}}$ then

$$
{ }^{t+\Delta t} C^{i j k l}=X_{* . a}^{\mathrm{e} i .} X_{* . b}^{\mathrm{e} j .} X_{* . c}^{\mathrm{e} k .} X_{* . d}^{\mathrm{e} l . t+\Delta t} \bar{C}^{a b c d}
$$

and if ${ }^{t+\Delta t} \mathbb{C}_{S}$ is the material fourth-order constitutive tensor such that ${ }^{t+\Delta t} \dot{\mathbf{S}}={ }^{t+\Delta t} \mathbb{C}_{S}:{ }^{t+\Delta t} \dot{\mathbf{A}}$ (with $\mathbf{S}$ the second Piola-Kirchhoff stress tensor and $\mathbf{A}$ the Green-Lagrange strain tensor), then

$$
{ }^{t+\Delta t} C_{S}^{i j k l}={ }_{t+\Delta t}^{0} X_{. a}^{\mathrm{p} i .}{ }_{t+\Delta t}^{0} X_{. b}^{\mathrm{p} j .}{ }_{t+\Delta t}^{0} X_{. c}^{\mathrm{p} k .}{ }_{t+\Delta t}^{0} X_{. d}^{\mathrm{p} l \cdot t+\Delta t} \bar{C}^{a b c d}
$$

The desired tensor depends on whether we are using the updated Lagrangian (UL) formulation or the total Lagrangian (TL) formulation.

We are now left only with the need for the computation of ${ }^{t+\Delta t} \overline{\mathbb{C}}$ defined by (102). We note that

$$
{ }^{t+\Delta t} \overline{\mathbf{S}}=\mathbf{X}_{*}^{\mathrm{e}-1 t+\Delta t} \tau \mathbf{X}_{*}^{\mathrm{e}-\mathrm{T}}
$$

For the case at hand with $\mathbf{Q}=\mathbf{R}_{*}^{\mathrm{eT}}$, using $\left(58_{1}\right)$ we are left with

$$
{ }^{t+\Delta t} \overline{\mathbf{S}}=\mathbf{U}_{*}^{\mathrm{e}-1 t+\Delta t} \bar{\tau} \mathbf{U}_{*}^{\mathrm{e}-1}
$$

The $\zeta$-derivative of ${ }^{t+\Delta t} \overline{\mathbf{S}}$ is written as

$$
{ }^{t+\Delta t} \dot{\overline{\mathbf{S}}}=\frac{\cdot}{\mathbf{U}_{*}^{\mathrm{e}-1}} t+\Delta t \bar{\tau} \mathbf{U}_{*}^{\mathrm{e}-1}+\mathbf{U}_{*}^{\mathrm{e}-1 t+\Delta t} \dot{\bar{\tau}} \mathbf{U}_{*}^{\mathrm{e}-1}+\mathbf{U}_{*}^{\mathrm{e}-1 t+\Delta t} \frac{\cdot}{\bar{\tau} \mathbf{U}_{*}^{\mathrm{e}-1}}
$$

Using

$$
\mathbf{\Omega}^{N *}:=\sum_{i=1}^{3} \dot{\mathbf{N}}_{i}^{*} \otimes \mathbf{N}_{i}^{*}=\sum_{i=1}^{3} \sum_{j=1}^{3} \Omega_{i j}^{N *} \mathbf{N}_{i}^{*} \otimes \mathbf{N}_{j}^{*}
$$

The derivative of the inverse of the trial stretch tensor is obtained in spectral form as

$$
\overline{\mathbf{U}_{*}^{\mathrm{e}-1}}=\sum_{i=1}^{3}-\frac{1}{\lambda_{i}^{* 2}} \dot{\lambda}_{i}^{*} \mathbf{N}_{i}^{*} \otimes \mathbf{N}_{i}^{*}+\sum_{i=1}^{3} \sum_{j \neq i}\left(\frac{1}{\lambda_{j}^{*}}-\frac{1}{\lambda_{i}^{*}}\right) \Omega_{i j}^{N *} \mathbf{N}_{i}^{*} \otimes \mathbf{N}_{j}^{*}
$$


whereas the derivative of the trial elastic Green-Lagrange strain is

$$
\dot{\overline{\mathbf{A}}}_{*}=\sum_{i=1}^{3} \lambda_{i}^{*} \dot{\lambda}_{i}^{*} \mathbf{N}_{i}^{*} \otimes \mathbf{N}_{i}^{*}+\sum_{i=1}^{3} \sum_{j \neq i} \frac{1}{2}\left(\lambda_{j}^{* 2}-\lambda_{i}^{* 2}\right) \Omega_{i j}^{N *} \mathbf{N}_{i}^{*} \otimes \mathbf{N}_{j}^{*}
$$

Therefore, using the customary arguments, the following geometric tensor $\mathbb{U}_{*}$ relates both rates as $\overline{\mathbf{U}_{*}^{\mathrm{e}-1}}=\mathbb{U}_{*}: \dot{\overline{\mathbf{A}}}_{*}$ :

$$
\mathbb{U}_{*}=\sum_{i=1}^{3}-\frac{1}{\lambda_{i}^{* 3}} \mathbf{M}_{i}^{*} \otimes \mathbf{M}_{i}^{*}+\sum_{i=1}^{3} \sum_{j \neq i} 2 \frac{1 / \lambda_{j}^{*}-1 / \lambda_{i}^{*}}{\lambda_{j}^{* 2}-\lambda_{i}^{* 2}} \mathbf{M}_{i}^{*} \stackrel{S}{\odot} \mathbf{M}_{j}^{*}
$$

where for short we defined (no sum on $i, j$ )

$$
\begin{aligned}
\mathbf{M}_{i}^{*} & :=\mathbf{N}_{i}^{*} \otimes \mathbf{N}_{i}^{*} \\
\mathbf{M}_{i}^{*} \stackrel{S}{\odot} \mathbf{M}_{j}^{*} & :=\frac{1}{4}\left(\mathbf{N}_{i}^{*} \otimes \mathbf{N}_{j}^{*}+\mathbf{N}_{j}^{*} \otimes \mathbf{N}_{i}^{*}\right) \otimes\left(\mathbf{N}_{i}^{*} \otimes \mathbf{N}_{j}^{*}+\mathbf{N}_{j}^{*} \otimes \mathbf{N}_{i}^{*}\right)
\end{aligned}
$$

The tensor $\mathbb{U}_{*}$ has minor and major symmetries and can, therefore, be stored in the commonly reduced form used for constitutive tensors. For the special case $\lambda_{i}^{*}=\lambda_{j}^{*}$ it is straightforward to show that

$$
\lim _{\lambda_{j}^{*} \rightarrow \lambda_{i}^{*}} 2 \frac{1 / \lambda_{j}^{*}-1 / \lambda_{i}^{*}}{\lambda_{j}^{* 2}-\lambda_{i .}^{* 2}}=-\frac{1}{\lambda_{i}^{* 3}}
$$

Hence (107) may be rewritten as

$$
{ }^{t+\Delta t} \dot{\overline{\mathbf{S}}}={ }^{t+\Delta t} \mathbb{T}: \dot{\overline{\mathbf{A}}}_{*}+\left(\mathbb{U}_{*}: \dot{\overline{\mathbf{A}}}_{*}\right):{ }^{t+\Delta t} \overline{\mathbf{Z}}+{ }^{t+\Delta t} \overline{\mathbf{Z}}^{\mathrm{T}}:\left(\mathbb{U}_{*}: \dot{\overline{\mathbf{A}}}_{*}\right)
$$

where the unsymmetric two-point stress tensor ${ }^{t+\Delta t} \overline{\mathbf{Z}}$ is defined as (note that $\bar{\tau}_{*}$ and $\mathbf{U}_{*}^{\mathrm{e}-1}$ commute in isotropic elasticity but ${ }^{t+\Delta t} \bar{\tau}$ and $\mathbf{U}_{*}^{\mathrm{e}-1}$ do not commute in general)

$$
{ }^{t+\Delta t} \overline{\mathbf{Z}}:={ }^{t+\Delta t} \bar{\tau} \mathbf{U}_{*}^{\mathrm{e}-1}
$$

and the fourth-order tensor ${ }^{t+\Delta t} \mathbb{T}$, yet to be determined, is defined such that

$$
\mathbf{U}_{*}^{\mathrm{e}-1 t+\Delta t} \dot{\bar{\tau}}_{*}^{\mathrm{e}-1}={ }^{t+\Delta t} \mathbb{\mathbb { T }}: \dot{\overline{\mathbf{A}}}_{*}
$$

From Equation (117), by a straightforward application of the chain rule, it is easily checked that

$$
{ }^{t+\Delta t} \mathbb{T}=\mathbb{J}_{*}:{ }^{t+\Delta t} \mathbb{D}: \mathbb{E}_{*}
$$

In this expression $\mathbb{J}_{*}$ is a geometric tensor based on the trial stretch that maps contravariant tensors from the rotated to the stress-free configuration:

$$
J_{*}=\mathbf{U}_{*}^{\mathrm{e}-1} \odot \mathbf{U}_{*}^{\mathrm{e}-1} \text {, i.e. }\left(J_{*}\right)_{. . k l}^{i j . .}=\frac{1}{2}\left(U_{*}^{\mathrm{e}-1}\right)_{. k}^{i}\left(U_{*}^{\mathrm{e}-1}\right)_{. l}^{j .}+\frac{1}{2}\left(U_{*}^{\mathrm{e}-1}\right)_{. l}^{i_{i}}\left(U_{*}^{\mathrm{e}-1}\right)_{. k}^{j .}
$$

where the lowering index operation is $\left(U^{\mathrm{e}-1}\right)^{i}{ }_{k}=\left(U^{\mathrm{e}-1}\right)^{i n} I_{n k}$ and $\mathbf{I}$ is the metric tensor (the identity in the Euclidean space used). The tensor $\mathbb{J}_{*}$ has also the minor symmetries that allow compact matrix storage. The tensor $\mathbb{E}_{*}$ in (118) is also a geometrical tensor based on trial 
measures, i.e. a fourth-order tensor such that $\dot{\mathbf{E}}_{*}^{\mathrm{e}}=\mathbb{E}_{*}: \dot{\overline{\mathbf{A}}}_{*}$. Using the spectral decomposition of $\mathbf{E}_{*}^{\mathrm{e}}$, the derivative is written as

$$
\dot{\mathbf{E}}_{*}^{\mathrm{e}}=\sum_{i=1}^{3} \frac{1}{\lambda_{i}^{*}} \dot{\lambda}_{i}^{*} \mathbf{N}_{i}^{*} \otimes \mathbf{N}_{i}^{*}+\sum_{i=1}^{3} \sum_{j \neq i}\left(\ln \lambda_{j}^{*}-\ln \lambda_{i}^{*}\right) \Omega_{i j}^{N *} \mathbf{N}_{i}^{*} \otimes \mathbf{N}_{j}^{*}
$$

and by $(110), \mathbb{E}_{*}$ is expressed in spectral form as

$$
\mathbb{E}_{*}=\sum_{i=1}^{3} \frac{1}{\lambda_{i}^{* 2}} \mathbf{M}_{i}^{*} \otimes \mathbf{M}_{i}^{*}+\sum_{i=1}^{3} \sum_{j \neq i} 2 \frac{\ln \lambda_{j}^{*}-\ln \lambda_{i}^{*}}{\lambda_{j}^{* 2}-\lambda_{i}^{* 2}} \mathbf{M}_{i}^{*} \stackrel{S}{\odot} \mathbf{M}_{j}^{*}
$$

In the special case of $\lambda_{i}^{*}=\lambda_{j}^{*}$ it is easily checked that

$$
\lim _{\lambda_{j}^{*} \rightarrow \lambda_{i}^{*}} 2 \frac{\ln \lambda_{j}^{*}-\ln \lambda_{i}^{*}}{\lambda_{j}^{* 2}-\lambda_{i}^{* 2}}=\frac{1}{\lambda_{i}^{* 2}}
$$

Finally, it is noted that in (118), the tensor ${ }^{t+\Delta t} \mathbb{D}$ relates the derivative of ${ }^{t+\Delta t} \bar{\tau}$ with that of $\mathbf{E}_{*}^{e}$ and is the only one in the computation of $\overline{\mathbb{C}}$ that is material-dependent. If this tensor is known the constitutive tensor in (102) is written in component form as

$$
\bar{C}^{i j k l}=\left(J_{*}\right)_{. . . m n}^{i j . .}\left({ }^{t+\Delta t} D\right)^{m n p q}\left(E_{*}\right)_{p q . .}^{. k l}+\left(U_{*}\right)^{i n k l}(\bar{Z})_{n .}^{. j}+(\bar{Z})_{n .}^{. i}\left(U_{*}\right)^{n j k l}
$$

which has also minor symmetries. The spatial or material tensor may be obtained through (103) or (104).

The tensor ${ }^{t+\Delta t} \mathbb{D}$ coincides with the constitutive tensor returned by the small strain plastic correction algorithm in the frequent assumption of no plastic spin $\left({ }^{t+\Delta t} \bar{\tau}={ }^{t+\Delta t} \overline{\mathbf{T}}\right)$. Otherwise, in the presence of plastic spin ${ }^{t+\Delta t} \mathbb{D}$ needs the following slightly more elaborate formulation. Taking the derivative of $\left(56_{2}\right)$ :

$$
\begin{aligned}
\dot{\bar{\tau}} & ={ }_{t}^{t+\Delta t}{ }_{t} \mathbf{R}^{w+\Delta t} \dot{\overline{\mathbf{T}}}^{t+\Delta t}{ }_{t} \mathbf{R}^{w \mathrm{~T}}+{ }^{t+\Delta t}{ }_{t} \dot{\mathbf{R}}^{w}{ }_{t}^{t+\Delta t}{ }_{t} \mathbf{R}^{w \mathrm{~T} t+\Delta t} \bar{\tau}+{ }^{t+\Delta t} \bar{\tau}_{\bar{\tau}}^{t+\Delta t}{ }_{t} \mathbf{R}^{w t+\Delta t} \dot{\mathrm{R}}^{w \mathrm{~T}} \\
& ={ }^{t+\Delta t}{ }_{t} \mathbf{R}^{w+\Delta t} \dot{\overline{\mathbf{T}}}^{t+\Delta t}{ }_{t} \mathbf{R}^{w \mathrm{~T}}+{ }^{t+\Delta t} \overline{\mathbf{W}}^{\mathrm{p} t+\Delta t} \overline{\bar{\tau}}-{ }^{t+\Delta t} \bar{\tau}^{t+\Delta t} \overline{\mathbf{W}}^{\mathrm{p}}
\end{aligned}
$$

Following similar steps as for expression (115) and defining

$$
\begin{aligned}
{ }^{t+\Delta t} \tilde{\mathbb{D}}_{w} & :=\mathbb{R}_{w}:{ }^{t+\Delta t} \tilde{\mathbb{D}} \\
\mathbb{R}_{w} & :={ }^{t+\Delta t}{ }_{t} \mathbf{R}^{w} \odot{ }^{t+\Delta t}{ }_{t} \mathbf{R}^{w}
\end{aligned}
$$

where ${ }^{t+\Delta t} \tilde{\mathbb{D}}$ is the unmodified small strain constitutive tensor that relates ${ }^{t+\Delta t} \dot{\overline{\mathbf{T}}}$ to $\dot{\mathbf{E}}_{*}^{\mathrm{e}}$ and the tensor ${ }^{t+\Delta t} \tilde{\mathbb{D}}_{w}$ relates ${ }^{t+\Delta t}{ }_{t} \mathbf{R}^{w} t+\Delta t \dot{\overline{\mathbf{T}}}^{t+\Delta t}{ }_{t} \mathbf{R}^{w \mathrm{~T}}$ with $\dot{\mathbf{E}}_{*}^{\mathrm{e}}$ (i.e. is the small strains constitutive tensor in the updated configuration). The tensor ${ }^{t+\Delta t} \mathbb{D}$ may be written in component form as

$$
{ }^{t+\Delta t} D^{i j k l}={ }^{t+\Delta t} \tilde{D}_{w}^{i j k l}+{ }^{t+\Delta t} W_{. n . .}^{i . k l}{ }^{t+\Delta t} \bar{\tau}^{n j}-{ }^{t+\Delta t} \bar{\tau}^{i n t+\Delta t} W_{n . .}^{. j k l}
$$

where ${ }^{t+\Delta t} \mathbb{W}$ is such that

$$
{ }^{t+\Delta t} \overline{\mathbf{W}}^{\mathrm{p}}={ }^{t+\Delta t} \mathbb{W}: \dot{\mathbf{E}}_{*}^{\mathrm{e}}
$$


which obviously depends on the constitutive equations and integration algorithm for the plastic spin. Again, ${ }^{t+\Delta t} \tilde{\mathbb{D}}_{w}, \mathbb{R}_{w}$ and $\left[{ }^{t+\Delta t} W_{. n . .}^{i . k l}{ }^{t+\Delta t} \bar{\tau}^{n j}-{ }^{t+\Delta t} \bar{\tau}^{i n}{ }^{t+\Delta t} W_{n \ldots}^{. j k l}\right]$ have minor symmetries and can be stored/operated on in compact format.

It it worth to mention that the previous constitutive tangent is tied to the assumption that the volumetric part of the stored energy function is of the form

$$
\mathscr{U}(J)=\frac{1}{2} \kappa(\ln J)^{2}
$$

where $\kappa$ is interpreted as the bulk modulus. The pressure for this function is $p=\kappa J^{-1} \ln J$. In deviatoric plasticity the flow is isochoric and it is necessary to resort to mixed formulations in order to avoid locking [32,48], see also Reference [49] for an assumed strain method. An unrestricted $u-p$ formulation that can be used for arbitrary $\mathscr{U}(J)$ expressions requires an evaluation of the Jacobian determinant $\tilde{J}$ corresponding to the separately interpolated pressure $\tilde{p}$. For the mentioned $\mathscr{U}(J)$, the inversion yields a Lambert $W$ function [50] of non-trivial evaluation. In order to avoid these computations, given that the elastic deformations are small, we can use

$$
\mathscr{U}(J)=\kappa[1+J(\ln J-1)]
$$

with the pressure $p=\kappa \ln J$. This function-in contrast with (130)-is convex, i.e. $\mathscr{U}^{\prime \prime}(J)=$ $\kappa / J>0$ and has the correct asymptotic values: $\mathscr{U}(1)=0, \mathscr{U}^{\prime}(1)=0, \mathscr{U}^{\prime \prime}(1)=\kappa, \mathscr{U}(J \rightarrow \infty) \rightarrow$ $\infty, \mathscr{U}^{\prime}(J \rightarrow \infty) \rightarrow \infty$. However, in the compression limit $\mathscr{U}(J \rightarrow 0) \rightarrow \kappa$, so the companion function $\mathscr{C}(J)=\mathscr{U}(1 / J)$ must be used in the large compression regime (which unfortunately also requires the evaluation of the Lambert $W$ function):

$$
\mathscr{C}(J)=\kappa\left[1-J^{-1}(\ln J+1)\right], \quad 0<J \leqslant 1
$$

This function is also convex in the compression regime since $\mathscr{C}^{\prime \prime}(J)=\kappa J^{-3}(1-2 \ln J)>0$ and has the limit values: $\mathscr{C}(1)=0, \mathscr{C}^{\prime}(1)=0, \mathscr{C}^{\prime \prime}(1)=\kappa, \mathscr{C}(J \rightarrow 0) \rightarrow \infty, \mathscr{C}^{\prime}(J \rightarrow 0) \rightarrow-\infty$, $\mathscr{C}^{\prime \prime}(J \rightarrow 0) \rightarrow \infty$.

Obviously, the use of an arbitrary $\mathscr{U}(J)$ requires that the volumetric part of the tangent be corrected. To perform such correction, we subtract the volumetric part from the small strains tangent ${ }^{t+\Delta t} \tilde{\mathbb{D}} \leftarrow{ }^{t+\Delta t} \tilde{\mathbb{D}}-\kappa \mathbf{I} \otimes \mathbf{I}$, use the deviatoric stresses in (116), and then correct in the UL formulation the final large strain tensor as:

$$
{ }^{t+\Delta t} \mathbb{C} \leftarrow{ }^{t+\Delta t} \mathbb{C}+J^{2} \mathscr{U}^{\prime \prime} \mathbf{I} \otimes \mathbf{I}+J \mathscr{U}^{\prime}(\mathbf{I} \otimes \mathbf{I}-2 \llbracket)
$$

and in the TL formulation as:

$$
{ }^{t+\Delta t} \mathbb{C}_{S} \leftarrow{ }^{t+\Delta t} \mathbb{C}_{S}+J^{2} \mathscr{U}^{\prime \prime} \mathbf{C}^{-1} \otimes \mathbf{C}^{-1}+J \mathscr{U}^{\prime}\left(\mathbf{C}^{-1} \otimes \mathbf{C}^{-1}-2 \mathbf{C}^{-1} \odot \mathbf{C}^{-1}\right)
$$

\subsection{Isotropic hardening and mixed hardening without and with plastic spin}

In the case of isotropic hardening and kinematic/mixed hardening with the stress integration performed in the principal stress space the constitutive tangent simplifies considerably. The algorithmic constitutive tangent for these special cases was obtained by Simó [22] who used the procedure of hyperelasticity formulated in principal stretches, see References [51,52]. 
In these cases the tangent is written as

$$
{ }^{t+\Delta t} \mathbb{C}^{(i)}=\sum_{i=1}^{3} \sum_{j=1}^{3}\left(\frac{\partial^{t+\Delta t} \tau_{i}}{\partial \ln \lambda_{j}^{*}}-2^{t+\Delta t} \tau_{i} \delta_{i j}\right) \mathbf{m}_{i}^{*} \otimes \mathbf{m}_{j}^{*}+\sum_{i=1}^{3} \sum_{j \neq i} 2 \frac{{ }^{t+\Delta t} \tau_{i} \lambda_{j}^{* 2}-{ }^{t+\Delta t} \tau_{j} \lambda_{i}^{* 2}}{\lambda_{i}^{* 2}-\lambda_{j}^{* 2}} \mathbf{m}_{i}^{*} \stackrel{S}{\odot} \mathbf{m}_{j}^{*}
$$

where $\mathbf{m}_{i}^{*}=\mathbf{n}_{i}^{*} \otimes \mathbf{n}_{i}^{*}$ are the spatial trial eigenbases and $\partial^{t+\Delta t} \tau_{i} / \partial \ln \lambda_{j}^{*}$ is the small strain algorithmic tangent in the reduced principal stress space. The special case $\lambda_{i}^{*}=\lambda_{j}^{*}$ is given by

$$
\lim _{\lambda_{j}^{*} \rightarrow \lambda_{i}^{*}} 2 \frac{{ }^{t+\Delta t} \tau_{i} \lambda_{j}^{* 2}-{ }^{t+\Delta t} \tau_{j} \lambda_{i}^{* 2}}{\lambda_{i}^{* 2}-\lambda_{j}^{* 2}}=\frac{\partial^{t+\Delta t} \tau_{j}}{\partial \ln \lambda_{j}^{*}}-\frac{\partial^{t+\Delta t} \tau_{i}}{\partial \ln \lambda_{j}^{*}}-2^{t+\Delta t} \tau_{i}
$$

However, the above equations are only valid if the stress integration is performed in the reduced space of the three principal values, i.e. using Assumptions 1 and 2 of Section 3.2. If only Assumption 1 is considered, the principal stress directions are kept frozen during the plastic return, and the stress integration should be performed in the full stress space since the principal directions of the back-stress are not the same as those of the stress. In such a case, the general expression derived in the previous subsection must be used. The only unknown in that expression is the tensor ${ }^{t+\Delta t} \mathbb{W}$ in (129) which depends on the constitutive relation for the plastic spin. The plastic spin for this particular case may be obtained from (68) and written as

$$
{ }^{t+\Delta t} \overline{\mathbf{W}}^{\mathrm{p}}=\sum_{i=1}^{3} \sum_{j \neq i}\left(1-\frac{{ }^{t+\Delta t} \Omega_{i j}^{\tilde{N}}}{\Omega_{i j}^{N *}}\right) \Omega_{i j}^{N *} \mathbf{N}_{i}^{*} \otimes \mathbf{N}_{j}^{*}
$$

Using the definition in (129) and (140) we arrive at

$$
{ }^{t+\Delta t} \mathbb{W}=\sum_{i=1}^{3} \sum_{j \neq i} \frac{1-{ }^{t+\Delta t} \Omega_{i j}^{\tilde{N}} / \Omega_{i j}^{N *}}{\ln \lambda_{j}^{*}-\ln \lambda_{i}^{*}} \mathbf{N}_{i}^{*} \otimes \mathbf{N}_{j}^{*} \otimes \frac{1}{2}\left(\mathbf{N}_{i}^{*} \otimes \mathbf{N}_{j}^{*}+\mathbf{N}_{j}^{*} \otimes \mathbf{N}_{i}^{*}\right)
$$

where ${ }^{t+\Delta t} \Omega_{i j}^{\tilde{N}} / \Omega_{i j}^{N *}$ is known since it can be extracted from the small strain constitutive tangent as follows. Consider the derivatives of the rotated stress tensor and the trial logarithmic strains (similar expressions apply for the spatial formulation):

$$
\begin{aligned}
\dot{\overline{\mathbf{T}}} & =\sum_{i=1}^{3}{ }^{t+\Delta t} \dot{\tau}_{i}{ }^{t+\Delta t} \tilde{\mathbf{N}}_{i} \otimes{ }^{t+\Delta t} \tilde{\mathbf{N}}_{i}+\sum_{i=1}^{3} \sum_{j \neq i}\left({ }^{t+\Delta t} \tau_{j}-{ }^{t+\Delta t} \tau_{i}\right)^{t+\Delta t} \Omega_{i j}^{\tilde{N}}{ }^{t+\Delta t} \tilde{\mathbf{N}}_{i} \otimes{ }^{t+\Delta t} \tilde{\mathbf{N}}_{j} \\
\dot{\mathbf{E}}_{*}^{\mathrm{e}} & =\sum_{i=1}^{3} \frac{\mathrm{d}}{\mathrm{d} \zeta}\left(\ln \lambda_{i}^{*}\right) \mathbf{N}_{i}^{*} \otimes \mathbf{N}_{i}^{*}+\sum_{i=1}^{3} \sum_{j \neq i}\left(\ln \lambda_{j}^{*}-\ln \lambda_{i}^{*}\right) \Omega_{i j}^{N *} \mathbf{N}_{i}^{*} \otimes \mathbf{N}_{j}^{*}
\end{aligned}
$$

The rotated $\zeta$-derivative of $\overline{\mathbf{T}}$ is

$$
{ }_{t}^{t+\Delta t} \mathbf{R}^{w}{ }^{t+\Delta t} \dot{\overline{\mathbf{T}}}{ }^{t+\Delta t}{ }_{t} \mathbf{R}^{w \mathrm{~T}}=\sum_{i=1}^{3}{ }^{t+\Delta t} \dot{\tau}_{i} \mathbf{N}_{i}^{*} \otimes \mathbf{N}_{i}^{*}+\sum_{i=1}^{3} \sum_{j \neq i}\left({ }^{t+\Delta t} \tau_{j}-{ }^{t+\Delta t} \tau_{i}\right){ }^{t+\Delta t} \Omega_{i j}^{\tilde{N}} \mathbf{N}_{i}^{*} \otimes \mathbf{N}_{j}^{*}
$$


The spectral form of the constitutive tangent in (126) that relates both expressions is

$$
{ }^{t+\Delta t} \tilde{\mathbb{D}}_{w}=\sum_{i=1}^{3} \sum_{j=1}^{3} \frac{\partial^{t+\Delta t} \tau_{i}}{\partial \ln \lambda_{j}^{*}} \mathbf{M}_{i}^{*} \otimes \mathbf{M}_{j}^{*}+\sum_{i=1}^{3} \sum_{j \neq i} \frac{{ }^{t+\Delta t} \tau_{j}-{ }^{t+\Delta t} \tau_{i}{ }^{t+\Delta t} \Omega_{i j}^{\tilde{N}}}{\ln \lambda_{j}^{*}-\ln \lambda_{i}^{*}} \mathbf{M}_{i}^{*} \stackrel{S}{\odot} \mathbf{M}_{j}^{*}
$$

Thus, taking the dot product with $\left(\mathbf{N}_{i}^{*} \otimes \mathbf{N}_{j}^{*}+\mathbf{N}_{j}^{*} \otimes \mathbf{N}_{i}^{*}\right)(i \neq j)$ we arrive at

$$
\frac{{ }^{t+\Delta t} \Omega_{i j}^{\tilde{N}}}{\Omega_{i j}^{N *}}=\frac{\ln \lambda_{j}^{*}-\ln \lambda_{i}^{*}}{{ }^{t+\Delta t} \tau_{j}-{ }^{t+\Delta t} \tau_{i}}{ }^{t+\Delta t}\left(\tilde{D}_{w}\right)_{i j}^{*}
$$

where for short

$$
{ }^{t+\Delta t}\left(\tilde{D}_{w}\right)_{i j}^{*}:=\frac{1}{2}\left(\mathbf{N}_{i}^{*} \otimes \mathbf{N}_{j}^{*}+\mathbf{N}_{j}^{*} \otimes \mathbf{N}_{i}^{*}\right):{ }^{t+\Delta t} \tilde{\mathbb{D}}_{w}:\left(\mathbf{N}_{i}^{*} \otimes \mathbf{N}_{j}^{*}+\mathbf{N}_{j}^{*} \otimes \mathbf{N}_{i}^{*}\right)
$$

The tensor ${ }^{t+\Delta t} \mathbb{W}$ does not have minor symmetry in the first indices due to the lack of symmetry of ${ }^{t+\Delta t} \overline{\mathbf{W}}^{\mathrm{p}}$, but since it is skew-symmetric in such indices, the tensor may still be stored in compact matrix format if the skew symmetry is accounted for during operations with the tensor. It is easy to see from Equations (128), (138) and (142) that the effect of ${ }^{t+\Delta t} \mathbb{W}$ on ${ }^{t+\Delta t} \tilde{\mathbb{D}}_{w}$ is to substitute the geometric part which contains terms in ${ }^{t+\Delta t} \Omega_{i j}{ }_{i j}^{\tilde{N}} / \Omega_{i j}^{N *}$ by terms with the final ${ }^{t+\Delta t} \Omega_{i j}^{\tilde{N}} / \Omega_{i j}^{N *}=1$.

\section{ILLUSTRATIVE SOLUTIONS}

We consider in this section some numerical examples to illustrate the concepts discussed above. In the first two examples we study the behaviour predicted for one finite element under isochoric stretching and simple shear. The third and fourth examples are oriented towards demonstrating the performance of the algorithms in some more complex situations. The results for four different models are shown:

1. Pure isotropic hardening, which we label (IH) and for which all formulations yield exactly the same results given that the principal directions do not change during the plastic flow.

2. Pure kinematic hardening with no plastic spin, $\overline{\mathbf{W}}^{\mathrm{p}}=\mathbf{0}$, which we label $(\mathbf{K H})$, i.e. the model given by Eterović and Bathe [21] for pure kinematic hardening.

3. Pure kinematic hardening with the plastic flow performed with the principal stress directions fixed, which we label (KH w/PS), i.e. with $\overline{\mathbf{W}}^{\mathrm{p}}$ given by (68) and integrated using the full stress space. In this formulation, which we also refer to as a 'model', we enforce Assumption 1 of Section 3.2, but not Assumption 2.

4. Pure kinematic hardening with the stress integration performed in the reduced space of the three principal stresses, which we label (KH in RS), i.e. the model by Simó [22], which inadvertently introduces a backstress rotation. In other words, in this 'model', we enforce Assumptions 1 and 2 of Section 3.2.

\subsection{Isochoric stretching}

As a first example consider the isochoric stretching example, already considered in Reference [21]. The problem is modelled by a bilinear plane strain element and the imposed deformation 
gradient is

$$
{ }_{0}^{t} \mathbf{X}=\left[\begin{array}{lll}
\lambda(t) & & \\
& 1 / \lambda(t) & \\
& & 1
\end{array}\right]
$$

The material constants are $\kappa=166.67 \mathrm{MPa}, \mu=76.92 \mathrm{MPa}, \sigma_{0}=0.75 \mathrm{MPa}$ (initial yield stress) $H_{l}=2.0 \mathrm{MPa}$ (linear hardening modulus). The predictions using either isotropic or kinematic hardening are shown in Figure 4. The results for all the above-mentioned models, as of course anticipated, are coincident.

Figure 5 shows the predictions when a stretching cycle is performed. As could be inferred, the three kinematic hardenings present exactly the same response since no rotation of the principal stress directions takes place.

\subsection{Simple shear}

As a second more revealing example we have selected a simple shear, four node finite element in which the deformation gradient is given by

$$
{ }_{0}^{t} \mathbf{X}=\left[\begin{array}{ccc}
1 & \gamma(t) & \\
& 1 & \\
& & 1
\end{array}\right]
$$

also considered in References [21,39] (a similar problem can be also found in Reference [22]). The material constants are the same as in the previous example. The analysis has been performed using the backward Euler scheme and the results are given for comparison purposes. For

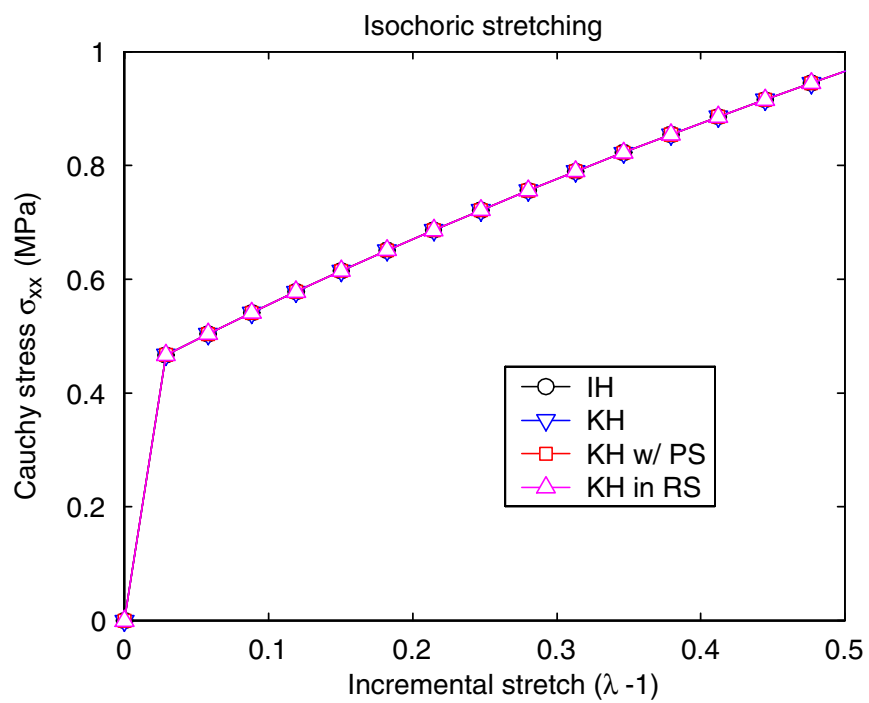

Figure 4. Predictions for the isochoric stretching example. 


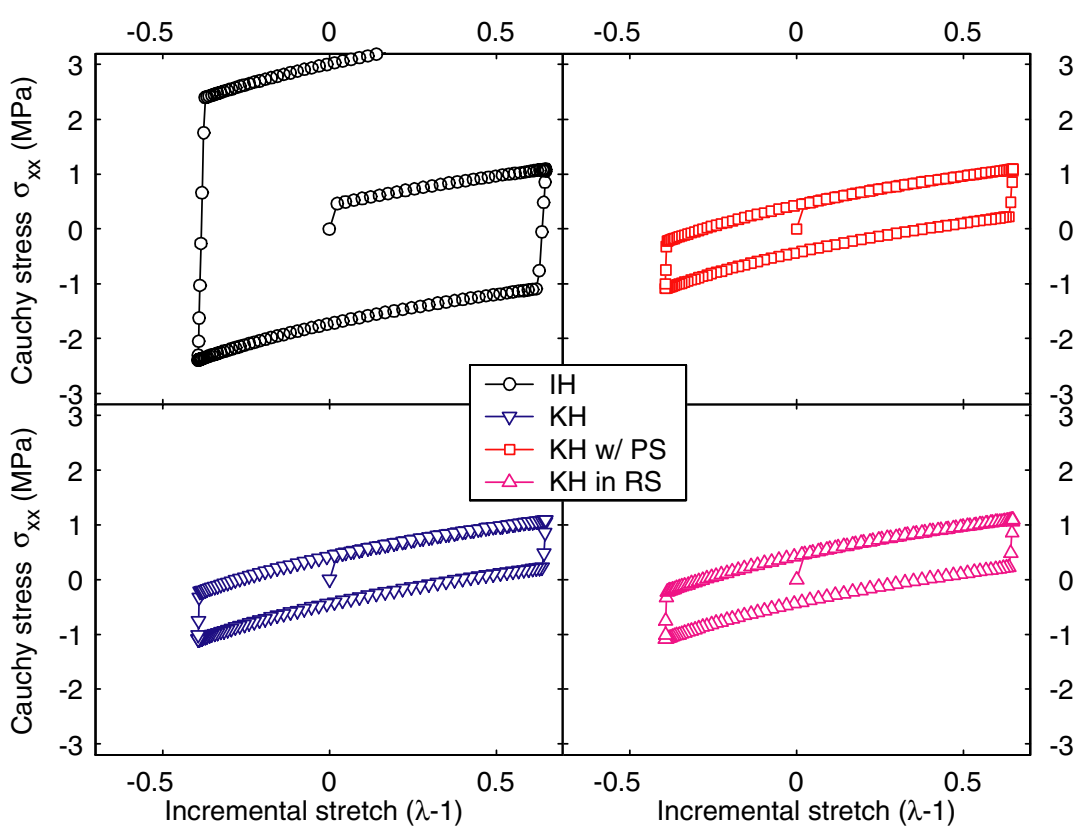

Figure 5. Predicted behaviour for isochoric stretching cycles.

a detailed analysis of the errors induced by the use of the backward Euler integration algorithm without plastic spin and results using the trapezoidal rule see Reference [31].

Figure 6 shows the results obtained. Except for the case of classical kinematic hardening (KH), the shear stress plots are almost identical for the range of deformations shown and essentially linear, as already noted in Reference [22]. In the case of kinematic hardening without plastic spin (KH) an oscillatory stress prediction is obtained, in contrast with the predictions using the other kinematic hardening models (KH w/PS) and (KH in RS). The presence of oscillatory response in kinematic hardening models is a well known fact also observed experimentally, see for example References [53-58] and references therein, and it is not only due to the use of the Jaumann rate as sometimes concluded. The inclusion of a plastic spin in kinematic hardening has been explored in the literature [53-58] in order to control the oscillatory response of this type of hardening. Of course, it is not our goal to elaborate on which model is more adequate for a specific material, but only to highlight the different predictions obtained. In fact, different results would have been obtained with other possible constitutive equations for the plastic spin and/or mixed hardening models.

The predictions of the other stresses are very different even for moderate deformations, as it can be seen in Figure 7 for the $\sigma_{y y}$ component. The basic kinematic hardening model (KH) presents in this component also an oscillatory behaviour as it is well known. The difference between the $(\mathbf{K H})$ model and the $(\mathbf{K H} \mathbf{w} / \mathbf{P S})$ model is only due to the plastic spin. It can be seen that there is also a difference between the response obtained using the kinematic hardening model with the plastic flow directions fixed using the full stress space (KW $\mathbf{W} / \mathbf{P S})$ and that using the reduced principal stress space (KH in RS). This difference can only be attributable 


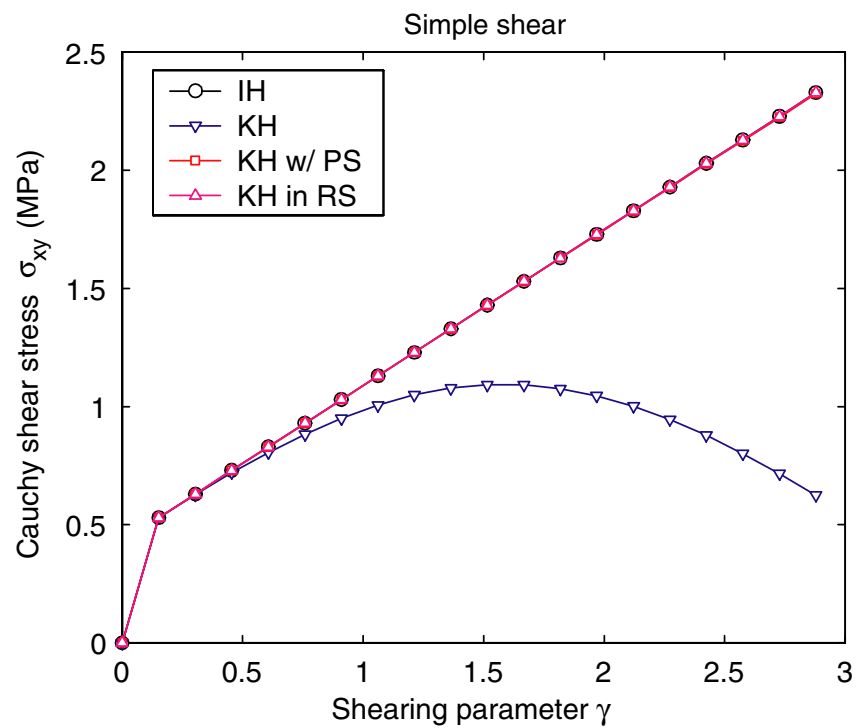

Figure 6. Predicted Cauchy shear stresses by the different hardening formulations for the case of simple shear monotonic loading.

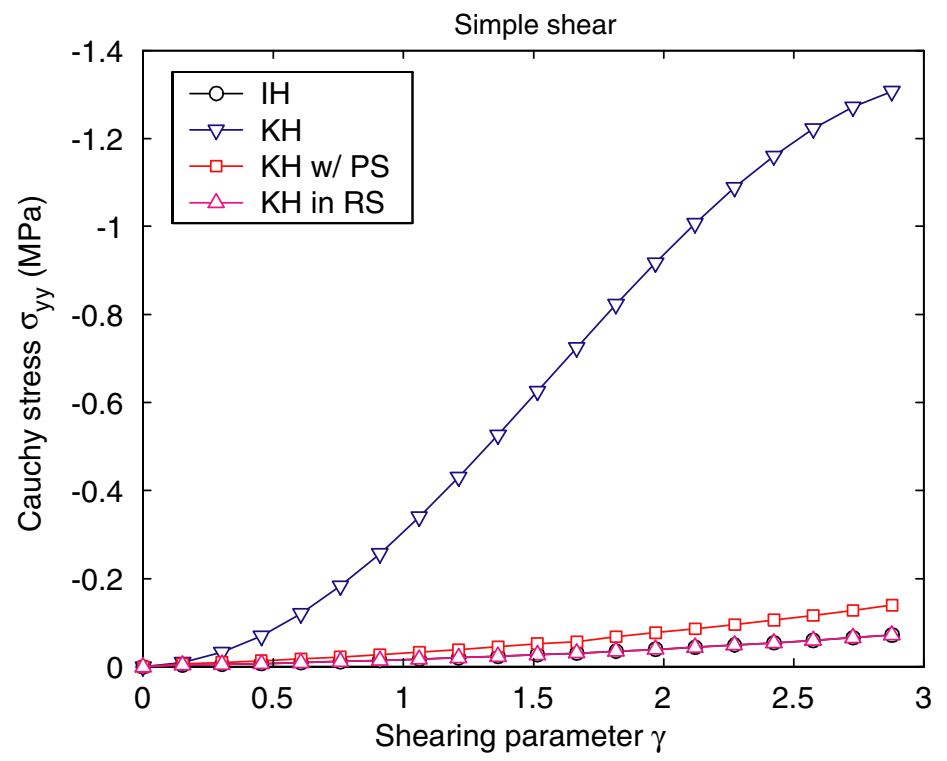

Figure 7. Predicted Cauchy normal stresses $\sigma_{y y}$ by the different hardening formulations for the case of simple shear monotonic loading.

to the effect of the different plastic rotations. Also note that the algorithm performed in the principal stress space (KH in RS) presents almost identical results to those using the isotropic hardening assumption. 
The cyclic behaviour of these models for a shearing cycle is shown in Figure 8 . It is somewhat surprising that under cyclic shearing the models (KH w/PS) and (KH in RS) give a cyclic behaviour close to the response obtained using isotropic hardening, and only the classical kinematic hardening model (KH) yields a behaviour with no growth of the hysteresis loop. To further study the influence of the hardening in cyclic plasticity, the same plots are calculated but with a larger plastic hardening modulus of $H_{l}=20 \mathrm{MPa}$, see Figure 9 . In this figure, it is seen that upon reversal, the models (KH w/PS) and (KH in RS) behave initially as the usual kinematic hardening model, but soon the plastic rotation orientates the flow so that the response becomes like we observe when using the isotropic hardening assumption. Of course, this is probably an undesirable behaviour if pure kinematic hardening is to be modelled. Thus, testing the cyclic behaviour is important in order to check the suitability of different plastic spin expressions, since to model cyclic loading is the actual motivation for using kinematic hardening models.

\subsection{Necking of a cylindrical bar}

The necking of a cylindrical bar has been studied for example in References [13,22], see also the references therein. Only a quarter of the specimen of length $53.334 \mathrm{~mm}$ and radius $6.413 \mathrm{~mm}$ is modelled due to symmetry conditions. The bulk and shear moduli are 164.2 and $80.19 \mathrm{GPa}$, respectively. The initial yield stress is $\tau_{0}=0.45 \mathrm{GPa}$. The hardening is modelled using the SPM method (see Reference [59]). The material loading function is given by the

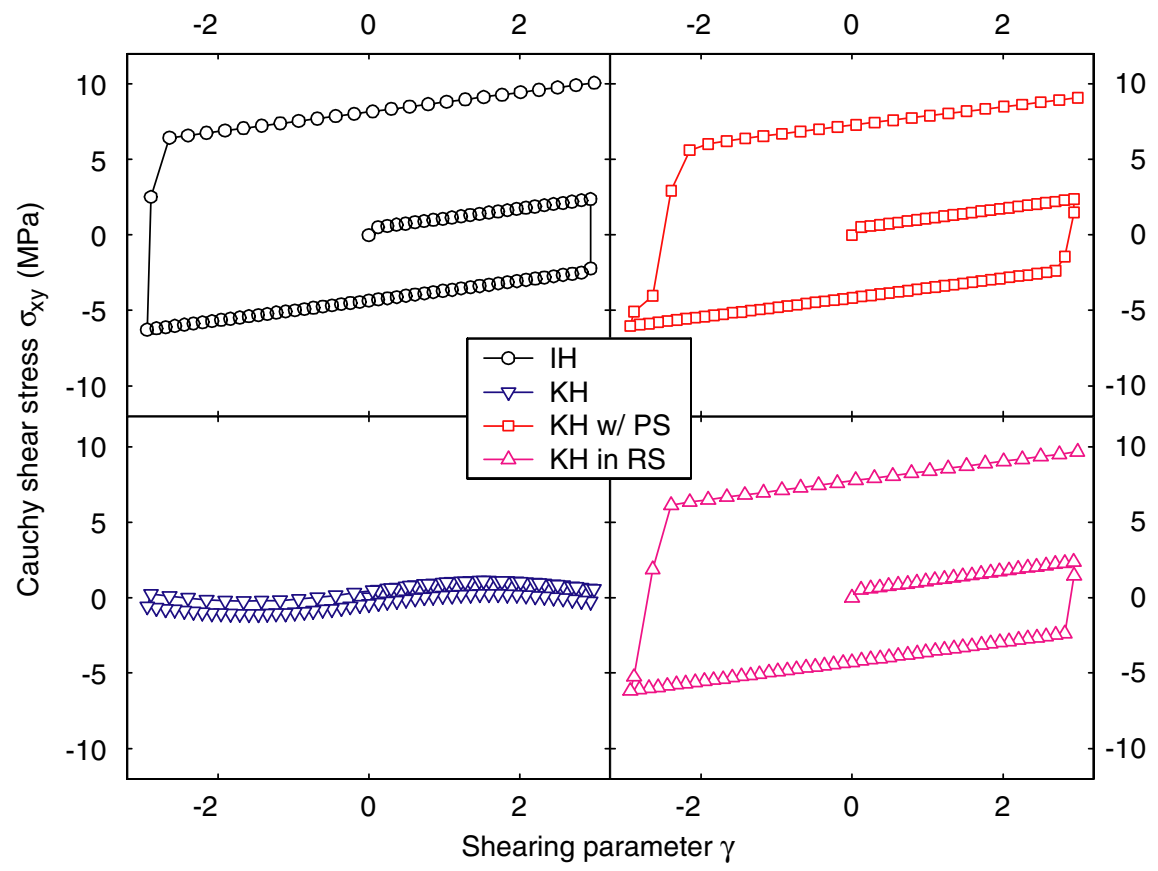

Figure 8. Predicted behaviour using the different hardening models for simple shear cyclic loading. Hardening modulus $H_{l}=2 \mathrm{MPa}$. 


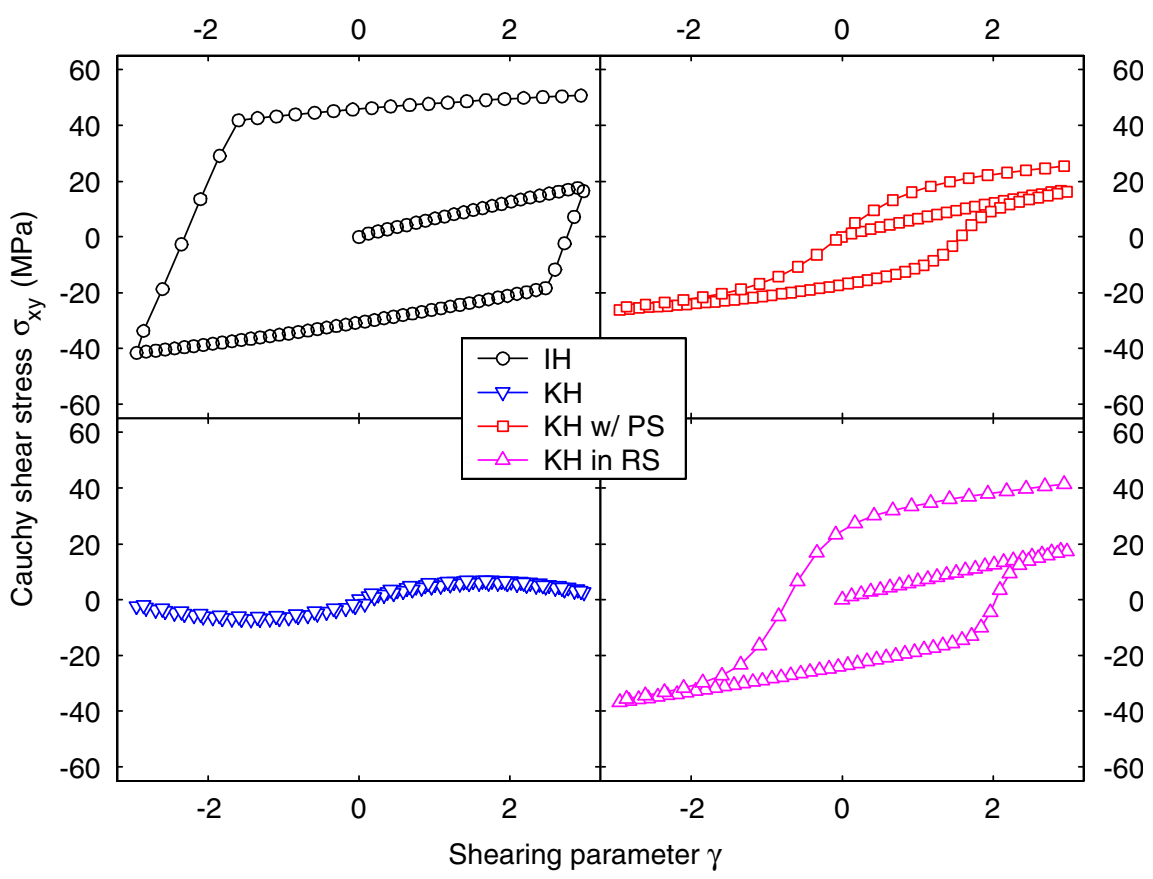

Figure 9. Predicted behaviour using the different hardening models for simple shear cyclic loading. Hardening modulus $H_{l}=20 \mathrm{MPa}$.

expression

$$
\hat{\tau}\left(\bar{E}^{\mathrm{p}}\right)=\tau_{0}+\left(\tau_{\infty}-\tau_{0}\right)\left[1-\exp \left(-\delta \bar{E}^{\mathrm{p}}\right)\right]+H_{l} \bar{E}^{\mathrm{p}}
$$

and the isotropic and kinematic hardening moduli are obtained, respectively, as

$$
\begin{aligned}
& K^{\prime}\left(\bar{E}^{\mathrm{p}}\right)=M \hat{\tau}^{\prime}\left(\bar{E}^{\mathrm{p}}\right) \\
& H^{\prime}\left(\bar{E}^{\mathrm{p}}\right)=(1-M) \hat{\tau}^{\prime}\left(\bar{E}^{\mathrm{p}}\right)
\end{aligned}
$$

In these expressions $\bar{E}^{\mathrm{p}}$ is the effective logarithmic plastic strain, $M$ is the mixed hardening parameter, $H_{l}$ is the linear hardening modulus, and $\tau_{\infty}, \delta$ are the saturation law material parameters. For the case at hand, these parameters are: $H_{l}=0.13 \mathrm{GPa}, \tau_{\infty}=0.715 \mathrm{GPa}$ and $\delta=16.93$. For the volumetric response equation (131) is used. The specimen is modelled using nine-noded mixed $(\mathbf{Q 9 / 3})$ axisymmetric elements (see Reference [32]). The mesh and the final configurations for isotropic hardening, classical kinematic hardening and kinematic hardening with flow in principal directions (KH in RS) are shown in Figure 10. The shading scale corresponds to equivalent logarithmic plastic strains. The neck radius reductions are presented in Figure 11.

As it could have been anticipated from the results of the previous example, the predictions using the isotropic hardening model (IH) and the kinematic hardening model in principal directions (KH in RS) are very similar-the $(\mathbf{K H} \mathbf{w} / \mathbf{P S})$ results are almost identical to those of (KH in RS) and thus not shown-, but different from those of the kinematic hardening 


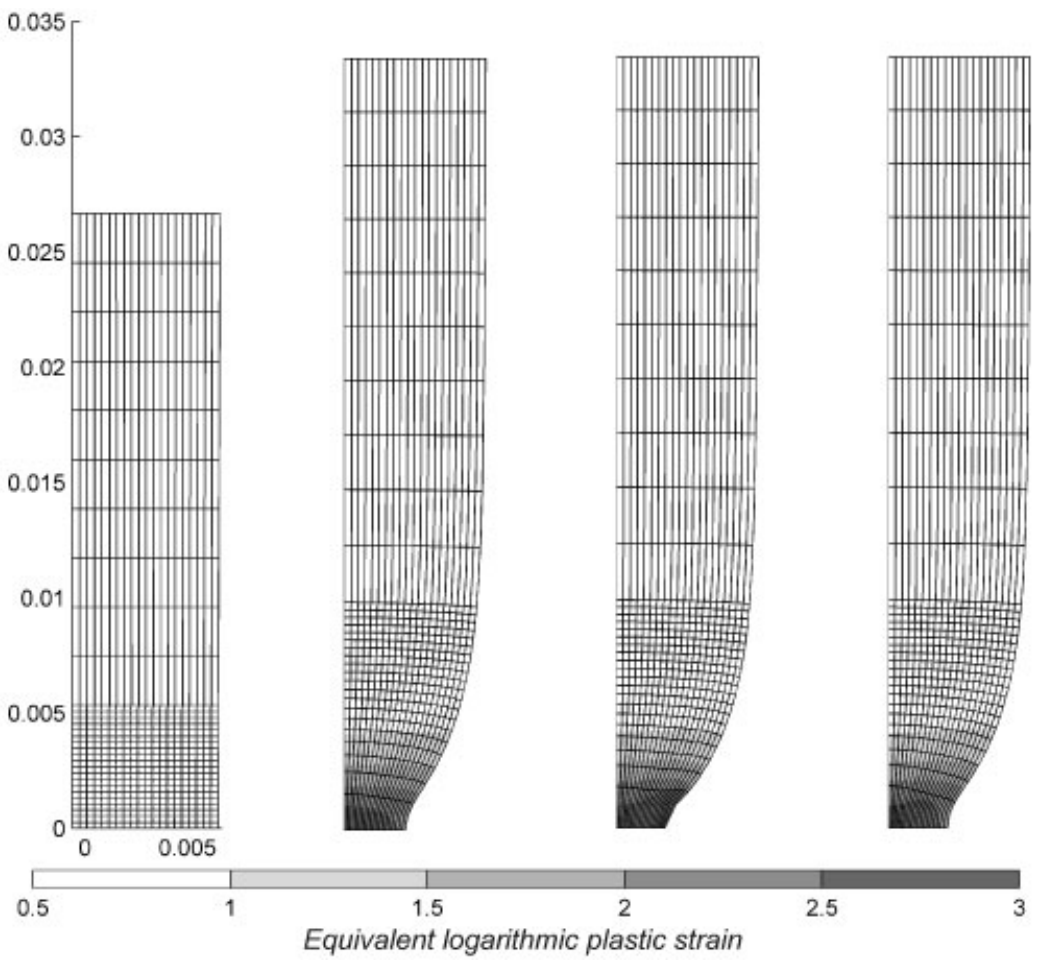

Figure 10. Necking of a circular bar. From left to right: Original mesh; final configurations and equivalent logarithmic plastic strains for isotropic hardening (IH), classical kinematic hardening (KH), kinematic hardening with flow in principal trial stress directions (KH in RS).

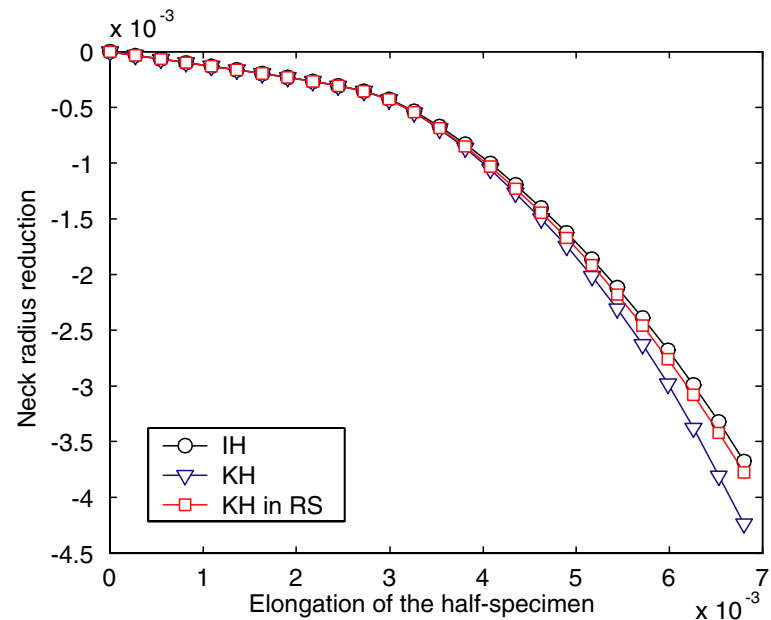

Figure 11. Necking of a circular bar: Neck radius reduction versus specimen elongation for the different hardening formulations. 


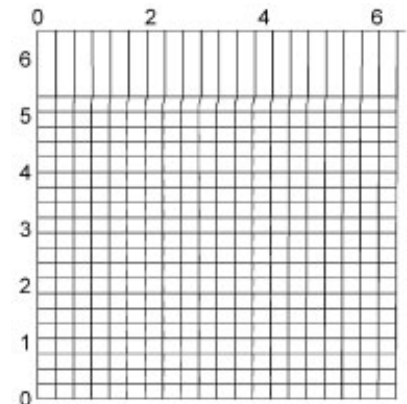

(a)

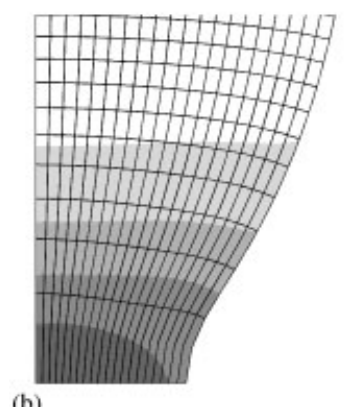

(b)

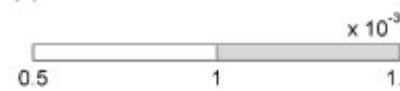

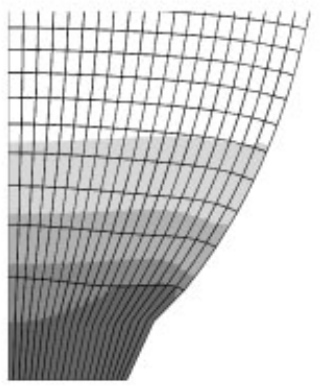

(c)

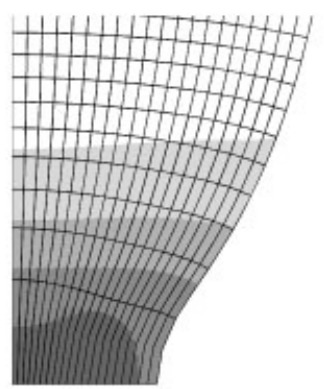

(d)

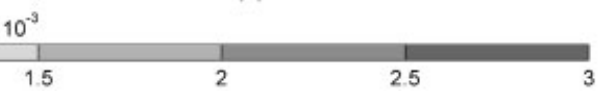

Figure 12. Necking of a circular bar: Close-up views of the necking zone: (a) original mesh; (b) isotropic hardening prediction (IH); (c) kinematic hardening prediction (KH); and (d) prediction with kinematic hardening flow in principal trial stresses (KH in RS).

model (KH), even though the stress change is almost proportional, and the necking effect is the only non-proportionality source. The rotation of the exterior part of the specimen in the necking zone induces a more pronounced plastic flow in that zone using the (KH) model, resulting in a larger necking effect. Figure 12 shows close-ups of the necking zones in the final configurations.

\subsection{Impact of a cylindrical bullet}

This problem, also considered in Reference [22], consists of the dynamic impact of a cylindrical bar against a rigid frictionless wall, is known as the Taylor impact test [60-62], and is used by many authors as a benchmark problem. The length of the specimen herein considered is $32.4 \mathrm{~mm}$ and the radius is $3.2 \mathrm{~mm}$. The elastic bulk modulus is $130 \mathrm{GPa}$ while the shear modulus is $40 \mathrm{GPa}$. The specimen has a density in the reference configuration of $\rho_{0}=8930 \mathrm{~kg} / \mathrm{m}^{3}$ and travels towards the wall at an initial speed of $227 \mathrm{~m} / \mathrm{s}$. The material hardens linearly with a modulus of $H_{l}=0.1 \mathrm{GPa}$. We modelled the specimen using 128 nine-noded mixed $(\mathbf{Q 9} / \mathbf{3})$ axisymmetric elements with a consistent mass matrix. Figure 13 shows the final configurations obtained with two of the hardening models together with the equivalent logarithmic plastic strains. It can be seen that the response predictions for this case are almost identical, a fact that can also be confirmed inspecting Figure 14, where the radius enlargement at the contact 


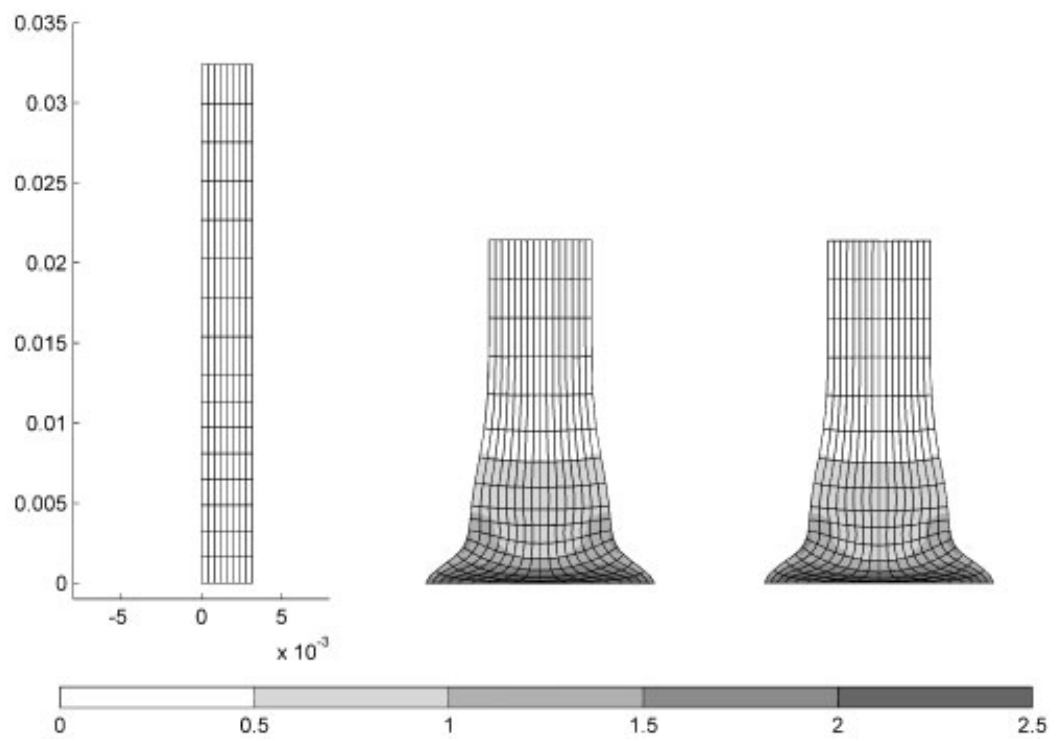

Figure 13. Impact of a cylindrical bullet: Original mesh (left) and final predicted configuration and equivalent logarithmic strains for isotropic (middle) hardening and kinematic hardening (right).

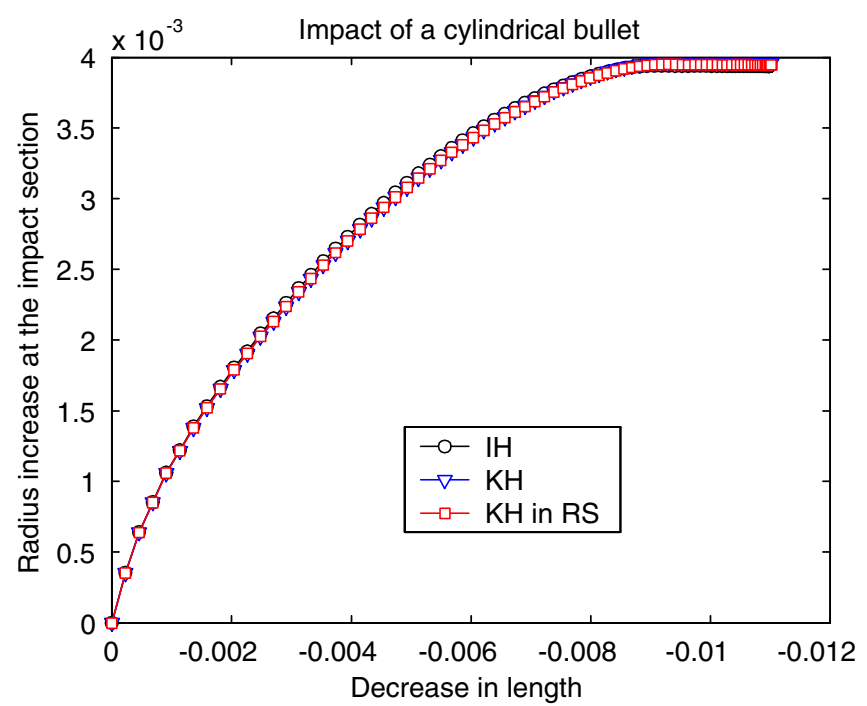

Figure 14. Impact of a cylindrical bullet: Radius increase at the impact section versus decrease in length for the different hardening models.

section is depicted. These results are in close agreement with those reported in Reference [22] and the predicted final state resembles the one obtained in experiments, cf. Figure 3.17 of Reference [63]. 


\section{CONCLUSIONS}

The formulation and an algorithm are presented for the analysis of large strain elasto-plastic problems incorporating mixed hardening and a possible constitutive equation for the plastic spin. The actual constitutive equation to be used is still to be explored [38]. The algorithm is based on the well known multiplicative decomposition of the deformation gradient. The consistent linearization of the stress integration algorithm is also presented.

This formulation allows to analyse in detail the algorithmic steps and solutions obtained using isotropic hardening and kinematic hardening with various assumptions. With the formulation given, two earlier published algorithms for kinematic hardening in large strain elasto-plastic analysis could be analysed, and it is shown that (inadvertently) a behaviour is obtained like when introducing a plastic spin. In simple shearing, the predicted response is like that obtained using an isotropic hardening model, but with little control on the actual physical behaviour.

It is clearly valuable to have an algorithm in which the plastic spin can be included in a physical manner. The formulation and algorithm given in this paper provide the basis for incorporating the plastic spin using an actual constitutive relation.

\section{ACKNOWLEDGEMENTS}

The present work has been carried out during a stay of the first author at the Massachusetts Institute of Technology, due to a leave granted by the Universidad de Castilla-La Mancha, which we gratefully acknowledge. Financial support for the major part of the stay was provided by the Secretaría de Estado de Educación y Universidades, Ministerio de Educación, Cultura y Deportes of Spain under Grant PR2003-0345. This is also part of the research project DPI2002-02065 (70\% FEDER funds) granted by the Secretaría de Estado de Política Científica y Tecnológica, Ministerio de Ciencia y Tecnología of Spain.

\section{REFERENCES}

1. Nagtegaal JC, Veldpaus FE. On the implementation of finite strain plasticity equations in a numerical model. In Numerical Analysis of Forming Processes, Pittman JFT, Zienkiewicz OC, Wood RD, Alexander JM (eds). Wiley: New York, 1984.

2. Goudreau GL, Hallquist JO. Recent developments in large-scale finite element Lagrangian hydrocode technology. Computer Methods in Applied Mechanics and Engineering 1982; 33:725-757.

3. Rolph III WD, Bathe KJ. On a large-strain finite-element formulation for elasto-plastic analysis. In Constitutive Equations, Macro and Computational Aspects, Willam KJ (ed.). Winter annual meeting. ASME: New York, 1984; 131-147.

4. Bathe KJ, Ramm E, Wilson EL. Finite element formulations for large deformation dynamic analysis. International Journal for Numerical Methods in Engineering 1975; 9:353-386.

5. Kojić M, Bathe KJ. Studies of finite element procedures-stress solution of a closed elastic strain path with stretching and shearing using the updated Lagrangian Jaumann formulation. Computers and Structures 1987; 26:175-179.

6. Eterović AL, Bathe KJ. A note on the use of the additive decomposition of the strain tensor in finite deformation inelasticity. Computer Methods in Applied Mechanics and Engineering 1991; 93:31-38.

7. Papadopoulus P, Lu J. A general framework for the numerical solution of problems in finite elasto-plasticity. Computer Methods in Applied Mechanics and Engineering 1998; 159:1-18.

8. Miehe C, Apel N, Lambrecht M. Anisotropic additive plasticity in the logarithmic strain space: modular kinematic formulation and implementation based on incremental minimization principles for standard materials. Computer Methods in Applied Mechanics and Engineering 2002; 191:5383-5426.

9. Lee EH. Elastic-plastic deformations at finite strains. Journal of Applied Mechanics (ASME) 1969; 36:1-6. 
10. Lee EH, Liu DT. Finite-strain elastic-plastic theory with application to plane wave analysis. Journal of Applied Physics 1967; 38:19-27.

11. Bilby BA, Bullough R, Smith E. Continuous distributions of dislocations: a new application of the methods of non-Riemannian geometry. Proceedings of the Royal Society of London Series A 1955; 231:263-273.

12. Asaro RJ. Micromechanics of crystals and polycrystals. Advances in Applied Mechanics 1983; 23:1-115.

13. Simó JC, Hughes TJR. Computational Inelasticity. Springer: New York, 1998.

14. Regueiro RA, Bammann DJ, Marín EB, Garikipati K. A nonlocal phenomenological anisotropic finite deformation plasticity model accounting for dislocation defects. Journal of Engineering Materials and Technology (ASME) 2002; 124:380-387.

15. Lubarda VA. Elastoplasticity Theory. CRC Press: Boca Ratón, FL, 2002.

16. Simó JC, Ortiz M. A unified approach to finite deformation elastoplasticity based on the use of hyperelastic constitutive equations. Computer Methods in Applied Mechanics and Engineering 1985; 49:221-245.

17. Simó JC. A framework for finite strain elastoplasticity based on maximum plastic dissipation and the multiplicative decomposition. Part I: continuum formulation. Computer Methods in Applied Mechanics and Engineering 1988; 66:199-219; Part II: computational aspects. Computer Methods in Applied Mechanics and Engineering 1988; 68:1-31.

18. Simó JC, Miehe C. Associative coupled thermoplasticity at finite strains: formulation, numerical analysis and implementation. Computer Methods in Applied Mechanics and Engineering 1992; 98:41-104.

19. Morán B, Ortiz M, Shih CF. Formulation of implicit finite element methods for multiplicative finite deformation plasticity. International Journal for Numerical Methods in Engineering 1990; 29:483-514.

20. Weber G, Anand L. Finite deformation constitutive equations and a time integration procedure for isotropic hyperelastic-viscoplastic solids. Computer Methods in Applied Mechanics and Engineering 1990; 79: $173-202$.

21. Eterović AL, Bathe KJ. A hyperelastic-based large strain elasto-plastic constitutive formulation with combined isotropic-kinematic hardening using the logarithmic stress and strain measures. International Journal for Numerical Methods in Engineering 1990; 30:1099-1114.

22. Simó JC. Algorithms for multiplicative plasticity that preserve the form of the return mappings of the infinitesimal theory. Computer Methods in Applied Mechanics and Engineering 1992; 99:61-112.

23. Perić D, de Souza EA. A new model for Tresca plasticity at finite strains with an optimal parametrization in the principal space. Computer Methods in Applied Mechanics and Engineering 1999; 171:463-489.

24. Cuitiño A, Ortiz M. A material-independent method for extending stress update algorithms from small strain plasticity to finite plasticity with multiplicative kinematics. Engineering Computations 1992; 9:437-451.

25. Perić D, Owen DRJ, Honnor ME. A model for finite strain elasto-plasticity based on logarithmic strains: computational issues. Computer Methods in Applied Mechanics and Engineering 1992; 94:35-61.

26. de Souza EA, Perić D. A computational framework for a class of fully coupled models for elastoplastic damage at finite strains with reference to the linearization aspects. Computer Methods in Applied Mechanics and Engineering 1996; 130:179-193.

27. Argyris JH, Doltsinis JSt. On the large strain inelastic analysis in natural formulation. Part I: quasistatic problems. Computer Methods in Applied Mechanics and Engineering 1979; 20:213-252.

28. Armero F. Formulation and finite element implementation of a multiplicative model of coupled poro-plasticity at finite strains under fully saturated conditions. Computer Methods in Applied Mechanics and Engineering 1999; 171:205-241.

29. Anand L. Moderate deformations in extension-torsion of incompressible isotropic elastic materials. Journal of the Mechanics and Physics of Solids 1986; 34:293-304.

30. Anand L. On H. Hencky's approximate strain-energy function for moderate deformations. Journal of Applied Mechanics (ASME) 1979; 46:78-82.

31. Gabriel G, Bathe KJ. Some computational issues in large strain elasto-plastic analysis. Computers and Structures 1995; 56:249-267.

32. Bathe KJ. Finite Element Procedures. Prentice-Hall: Englewood Cliffs, NJ, 1996.

33. Kojić M, Bathe KJ. Inelastic Analysis of Solids and Structures. Springer: New York, 2005.

34. Eidel B, Gruttmann F. On the theory and numerics of orthotropic elastoplasticity at finite plastic strains. In Computational Fluid and Solid Mechanics 2003, Bathe KJ (ed.). Elsevier: Oxford, 2003; 246-248.

35. Anand L. Constitutive equations for hot-working of metals. International Journal of Plasticity 1985; 1: $213-231$.

36. Simó JC. On the computational significance of the intermediate configuration and hyperelastic relations in finite deformation elastoplasticity. Mechanics of Materials 1986; 4:439-451. 
37. Mandel J. Thermodynamics and plasticity. In Foundations of Continuum Thermodynamics, Delgado JJ, Nina NR, Whitelaw JH (eds). Macmillan: London, 1974; 283-304.

38. Montáns FJ, Bathe KJ. A framework for computational large strain plasticity-anisotropic elasticity, anisotropic yield functions and mixed hardening, in preparation.

39. Montáns FJ, Bathe KJ. On the stress integration in large strain elastoplasticity. In Computational Fluid and Solid Mechanics 2003, Bathe KJ (ed.). Elsevier: Oxford, 2003; 494-497.

40. Anand L. Elasto-viscoplasticity: constitutive modelling and deformation processing. In Large Plastic Deformations, Teodosiu C, Raphanel JL, Sidoroff F (eds). Balkema: Rotterdam, 1993.

41. Von Houtte P. Anisotropic plasticity. In Numerical Modelling of Material Deformation Processes, Hartley P, Pillinger I, Sturgess C (eds). Springer: London, 1992.

42. Kowalewsky ZL, Śliwowsky M. Effect of cyclic loading on the yield surface evolution of 18G2A low-alloy steel. International Journal of Mechanical Sciences 1997; 39:51-68.

43. Montáns FJ. Implicit multilayer J2-plasticity using Prager's translation rule. International Journal for Numerical Methods in Engineering 2001; 50:347-376.

44. Montáns FJ. Implicit plane stress algorithm for multilayer J2-plasticity using the Prager-Ziegler translation rule. International Journal for Numerical Methods in Engineering 2004; 59:409-418.

45. Montáns FJ, Borja RI. Implicit J2-bounding surface plasticity using Prager's translation rule. International Journal for Numerical Methods in Engineering 2002; 55:1129-1166.

46. Kojić M, Bathe KJ. The effective-stress-function algorithm for thermo-elasto-plasticity and creep. International Journal for Numerical Methods in Engineering 1987; 24:1509-1532.

47. Miehe C. Numerical computation of algorithmic (consistent) tangent moduli in large-strain computational inelasticity. Computer Methods in Applied Mechanics and Engineering 1996; 134:223-240.

48. Sussman T, Bathe KJ. A finite element formulation for nonlinear incompressible elastic and inelastic analysis. Computers and Structures 1987; 26:57-409.

49. Simó JC, Armero F. Geometrically nonlinear enhanced strain mixed methods and the method of incompatible modes. International Journal for Numerical Methods in Engineering 1992; 33:1413-1449.

50. Corless RM, Gonnet GH, Hare DEG, Jeffrey DJ, Knuth DE. On the Lambert W Function. Advances in Computational Mathematics 1996; 5:329-359.

51. Simó JC, Taylor RL. Quasi-incompressible finite elasticity in principal stretches: Continuum basis and numerical algorithms. Computer Methods in Applied Mechanics and Engineering 1991; 85:273-310.

52. Ogden RW. Nonlinear Elastic Deformations. Ellis Horwood: Chichister, 1984.

53. Khan AS, Huang S. Continuum Theory of Plasticity. Wiley: New York, 1995.

54. Loret B. On the effects of plastic rotation in the finite deformation of anisotropic elastoplastic materials. Mechanics of Materials 1983; 2:287-304.

55. Dafalias YF. The plastic spin. Journal of Applied Mechanics (ASME) 1985; 52:865-871.

56. Dafalias YF. On the microscopic origin of the plastic spin. Acta Mechanica 1990; 82:31-48.

57. Kuroda M, Tvergaard V. Plastic spin associated with a non-normality theory of plasticity. European Journal of Mechanics - A/Solids 2001; 20:893-905.

58. Dafalias YF. The plastic spin: necessity or redundancy?. International Journal of Plasticity 1998; 14: 909-931.

59. Bathe KJ, Montáns FJ. On modelling mixed hardening in computational plasticity. Computers and Structures 2004; 82(6):535-539.

60. Taylor GI. The testing of materials at high rates of loading. Journal of the Institution of Civil Engineering 1946; 26:486-519.

61. Taylor GI. The use of flat ended projectiles for determining yield stress, I: theoretical considerations. Proceedings of the Royal Society of London Series A 1948; 194:289-299.

62. Whiffin AC. The use of flat ended projectiles for determining yield stress, II: tests on various metallic materials. Proceedings of the Royal Society of London Series A 1948; 194:300-322.

63. Meyers MA. Dynamic Behavior of Materials. Wiley: New York, 1994. 